Fürstenau, Bärbel; Hommel, Mandy; Förster, Manuel; Kraitzek, Andreas; Wuttke, Eveline; Aprea, Carmela; Rudeloff, Michelle; Siegfried, Christin

\title{
Messung von Finanzkompetenz - Ergebnisse eines Symposiums
}

Wittmann, Eveline [Hrsg.]; Frommberger, Dietmar [Hrsg.]; Weyland, Ulrike [Hrsg.]: Jahrbuch der berufs- und wirtschaftspädagogischen Forschung 2020. Opladen; Berlin; Toronto : Verlag Barbara Budrich 2020, S. 33-64. (Schriftenreihe der Sektion Berufs- und Wirtschaftspädagogik der Deutschen Gesellschaft für Erziehungswissenschaft (DGfE))

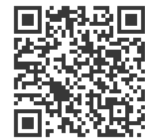

Quellenangabe/ Reference:

Fürstenau, Bärbel; Hommel, Mandy; Förster, Manuel; Kraitzek, Andreas; Wuttke, Eveline; Aprea, Carmela; Rudeloff, Michelle; Siegfried, Christin: Messung von Finanzkompetenz - Ergebnisse eines Symposiums - In: Wittmann, Eveline [Hrsg.]; Frommberger, Dietmar [Hrsg.]; Weyland, Ulrike [Hrsg.]: Jahrbuch der berufs- und wirtschaftspädagogischen Forschung 2020. Opladen; Berlin; Toronto : Verlag Barbara Budrich 2020, S. 33-64 - URN: urn:nbn:de:0111-pedocs-206549 - DOI: 10.25656/01:20654

https://nbn-resolving.org/urn:nbn:de:0111-pedocs-206549 https://doi.org/10.25656/01:20654

in Kooperation mit / in cooperation with:

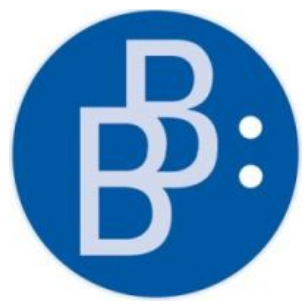

https://www.budrich.de

\section{Nutzungsbedingungen}

Dieses Dokument steht unter folgender Creative Commons-Lizenz: http://creativecommons.org/licenses/by-sa/4.0/deed.de - Sie dürfen das Werk bzw. den Inhalt vervielfältigen, verbreiten und öffentlich zugänglich machen sowie Abwandlungen und Bearbeitungen des Werkes bzw. Inhaltes anfertigen, solange sie den Namen des Autors/Rechteinhabers in der von ihm festgelegten Weise nennen und die daraufhin neu entstandenen Werke bzw. Inhalte nur unter Verwendung von Lizenzbedingungen weitergeben, die mit denen dieses Lizenzvertrags identisch, vergleichbar oder kompatibel sind.

Mit der Verwendung dieses Dokuments erkennen Sie die Nutzungsbedingungen an.

\section{Terms of use}

This document is published under following Creative Commons-License: http://creativecommons.org/licenses/by-sa/4.0/deed.en - You may copy, distribute and transmit, adapt or exhibit the work or its contents in public and alter, transform, or change this work as long as you attribute the work in the manner specified by the author or licensor. New resulting works or contents must be distributed pursuant to this license or an identical or comparable license.

By using this particular document, you accept the above-stated conditions of use.

\section{(†) (?}

\section{Kontakt / Contact:}

\section{peDOCS}

DIPF | Leibniz-Institut für Bildungsforschung und Bildungsinformation Informationszentrum (IZ) Bildung

E-Mail: pedocs@dipf.de

Internet: www.pedocs.de

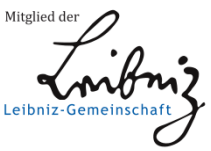




\section{Jahrbuch der berufs- und wirtschaftspädagogischen Forschung 2020}

Eveline Wittmann, Dietmar Frommberger, Ulrike Weyland (Hrsg.)

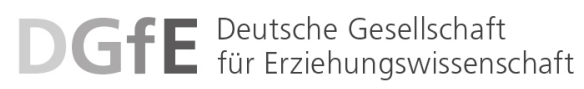


Jahrbuch der berufs- und

wirtschaftspädagogischen Forschung 2020 
Schriftenreihe der Sektion

Berufs- und Wirtschaftspädagogik

der Deutschen Gesellschaft für

Erziehungswissenschaft (DGfE) 
Eveline Wittmann

Dietmar Frommberger

Ulrike Weyland (Hrsg.)

Jahrbuch der berufs- und

wirtschaftspädagogischen

Forschung 2020

Verlag Barbara Budrich

Opladen • Berlin • Toronto 2020 
Bibliografische Information der Deutschen Nationalbibliothek

Die Deutsche Nationalbibliothek verzeichnet diese Publikation in der Deutschen

Nationalbibliografie; detaillierte bibliografische Daten sind im Internet über

http://dnb.d-nb.de abrufbar.

\section{Veröffentlicht mit Unterstützung der Käthe und Ulrich Pleiß-Stiftung}

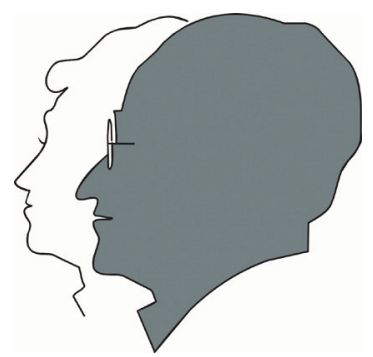

(C) 2020 Dieses Werk ist bei der Verlag Barbara Budrich GmbH erschienen und steht unter der Creative Commons Lizenz Attribution-ShareAlike 4.0 International (CC BY-SA 4.0): https://creativecommons.org/licenses/by-sa/4.0/.

Diese Lizenz erlaubt die Verbreitung, Speicherung, Vervielfältigung und Bearbeitung bei Verwendung der gleichen CC-BY-SA 4.0-Lizenz und unter Angabe der UrheberInnen, Rechte, Änderungen und verwendeten Lizenz.

www.budrich.de

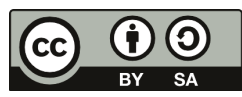

Dieses Buch steht im Open-Access-Bereich der Verlagsseite zum kostenlosen Download bereit (https://doi.org/10.3224/84742437).

Eine kostenpflichtige Druckversion (Print on Demand) kann über den Verlag bezogen werden. Die Seitenzahlen in der Druck- und Onlineversion sind identisch.

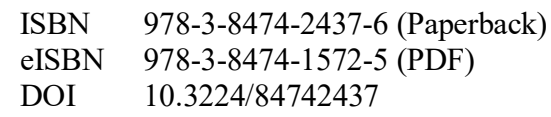

Umschlaggestaltung: Bettina Lehfeldt, Kleinmachnow - www.lehfeldtgraphic.de Druck: docupoint GmbH, Barleben

Printed in Europe 


\section{Inhaltsverzeichnis}

Vorwort: Forschungserträge und Forschungsperspektiven der Berufs- und Wirtschaftspädagogik

\section{Teil I: Reflexion der Disziplin: Außenperspektive}

Andrea Sailer

Literarische Zusammenschau zu den Tagungsbeiträgen

\section{Teil II: Erträge aus den Forschungszusammenhängen der Berufs- und Wirtschaftspädagogik: Finanzkompetenz und Bildung für nachhaltige Entwicklung}

Bärbel Fürstenau, Mandy Hommel, Manuel Förster, Andreas Kraitzek, Eveline Wuttke, Carmela Aprea, Michelle Rudeloff und Christin Siegfried Messung von Finanzkompetenz - Ergebnisse eines Symposiums

Andreas Fischer, Marc Casper, Karina Kiepe, Harald Hantke, Jan Pranger und Sören Schütt-Sayed

Theoretische Reflexionen zur Didaktik der Berufsbildung für nachhaltige Entwicklung (BBNE) aus der Perspektive der Modellversuchsforschung

\section{Teil III: (Zwischen)Ergebnisse aus Projekten}

\section{Berufsverbleib und Berufsorientierung}

Dana Bergmann

Verarbeitung von Studienabbrüchen innerhalb beruflicher Entwicklungsprozesse - eine berufsbiografische Analyse

Silke Lange

Vorzeitige Vertragslösungen aus der Perspektive der Auszubildenden ein Vorschlag für einen subjektorientierten Erklärungsansatz

Leonie Bogaczyk, Marie Schröder, Thomas Retzmann und Thomas Bienengräber

Theoretische Grundlagen und empirische Befunde zum Wissensmanagement in der inklusiven Berufsorientierung 
Heike Jahncke, Florian Berding, Jane Porath und Christian Steib Berufsdarstellungen in Vorabendserien zwischen 1990 und 2018 als Informationsquellen über Berufe

\section{Berufliche Didaktik und Curriculum}

Susanne Korth, Svenja Noichl und Volker Rexing

Inklusive Lernumgebungen mit digitalen Medien in Überbetrieblichen

Berufsbildungsstätten der Bauindustrie

Jutta Mohr, Isabelle Riedlinger und Karin Reiber

Die Bedeutung der Digitalisierung in der Neuausrichtung der pflegerischen Ausbildung - Herausforderungen für die berufliche Pflege im Kontext der Fachkräftesicherung

\section{Professionalisierung von Lehrkräften beruflicher Schulen}

Andrea Burda-Zoyke und Immo Degner

Erwartungen an Praxissemester im Studium für ein Lehramt an

beruflichen Schulen - eine qualitative Studie im Rahmen der

Studiengangentwicklung

Josephine Berger und Birgit Ziegler

Studienzufriedenheit und Studienerfolg im ersten Studienjahr -

Studierende im Lehramt an beruflichen Schulen und an Gymnasien

im Vergleich

\section{Teil IV: Reflexion der Disziplin: Innenperspektive}

Silke Lange, Dietmar Frommberger, Ulrike Weyland und

Eveline Wittmann

Die Qualitätsoffensive Lehrerbildung aus der Perspektive der

beruflichen Lehrerbildung

Herausgeberschaft

Autorinnen und Autoren 


\section{Messung von Finanzkompetenz - Ergebnisse eines Symposiums}

Bärbel Fürstenau, Mandy Hommel, Manuel Förster, Andreas Kraitzek, Eveline Wuttke, Carmela Aprea, Michelle Rudeloff und Christin Siegfried

\section{Hinführung und Ziel}

In den letzten Jahren wird eine wachsende Bedeutung von Financial Literacy diskutiert, nicht zuletzt in Folge von Wirtschafts-, Finanz- und Schuldenkrisen vieler Länder, einer zunehmenden privaten Verschuldung und der Tatsache, dass aufgrund eines zunehmenden Rückzugs vieler Staaten aus sozialen Sicherungssystemen den Individuen mehr Verantwortung für finanzielle Angelegenheiten zukommt (Aprea et al. 2016). Aktuellen empirischen Studien zufolge weist in vielen Ländern ein großer Teil der Bevölkerung Defizite sogar in grundlegenden finanziellen Kenntnissen auf (OECD 2017b).

Angesichts dieser Situation ist Financial Literacy bzw. Finanzkompetenz ${ }^{1}$, d. h. die Fähigkeit, mit Geld und finanziellen Belangen angemessen umzugehen, für das tägliche Leben und für das langfristige finanzielle Wohlergehen sowohl von Individuen als auch von Volkswirtschaften essenziell. Die Förderung dieser Kompetenz kann durch formale Bildungsbemühungen oder informelle Lerngelegenheiten unterstützt werden. Voraussetzung dafür ist eine angemessene Modellierung und vor allem Messung dieser Kompetenz. Auf dieser Basis können zielgruppengerechte Interventionen gestaltet werden.

Derzeitige Messverfahren fokussieren jeweils Teile einer umfassenden Finanzkompetenz. Häufig steht die kognitive Komponente, speziell das Faktenwissen im Zentrum des Interesses, das über Multiple- oder Single-ChoiceItems mit einfachen Antwortstämmen erfragt wird. Ein Forschungsdefizit ist in Messverfahren zu sehen, die situativ verankert und/oder auf bestimmte Domänen bezogen sind, die zudem nicht-kognitive Aspekte und neben Ergebnissen auch Prozesse in den Blick nehmen.

1 Angemerkt sei, dass in diesem Beitrag teils von Finanzkompetenz, teils von Financial Literacy die Rede ist. Dies ist unterschiedlichen Zugängen und sprachlichen Präferenzen geschuldet. Im Rahmen des Symposiums werden beide Begriffe synonym verwendet. Im Sinne eines funktionalen Verständnisses (Weinert 2001, 2002) beschreiben sie hier komplexe domänenspezifische Fähigkeiten, die mit der Bewältigung von Anwendungssituationen verbunden, sowohl kognitive als auch emotional-motivationale, volitionale und soziale Aspekte umfassen und durch Lernen und Erfahrungen erweitert und vertieft werden können. 
Vor diesem Hintergrund war es Ziel eines Symposiums der Jahrestagung der Sektion Berufs- und Wirtschaftspädagogik 2019, zunächst einen Überblick über bestehende Instrumente und Möglichkeiten der Erfassung von Finanzkompetenz zu gewinnen und die Messung von Finanzkompetenz anschließend an zwei Beispielen zu verdeutlichen: Der Beitrag von Förster und Kraitzek (Abschnitt 2) gibt einen Überblick darüber, welche nationalen und internationalen Messverfahren vorhanden sind. Aufbauend darauf schließen die Autoren eine Forschungslücke, indem sie Desiderata vorhandener Messverfahren aufzeigen und indem sie ausloten, welche Richtung in Bezug auf Messung von Finanzkompetenz zukünftig eingeschlagen werden kann oder sollte. Der Beitrag von Wuttke, Siegfried und Aprea (Abschnitt 3) macht deutlich, wie verschiedene Facetten der Finanzkompetenz mit Situational Judgement Tests (SJT) an Stelle von Einfach- oder Mehrfachauswahlaufgaben erfasst werden können und wie bestimmte Gruppen (unterteilt nach Geschlecht, Bildungsund Migrationshintergrund) dabei abschneiden. Die Autorinnen schließen damit eine Lücke zur Klärung der Testergebnisse und Gruppenunterschiede in Abhängigkeit vom eingesetzten Instrument. Der Beitrag von Hommel und Fürstenau (Abschnitt 4) widmet sich dem komplexen Finanzprodukt der Baufinanzierung und zeigt auf, inwiefern informelles Lernen unter Berücksichtigung von Online-Informationen zur Entwicklung dieser produktspezifischen Finanzkompetenz beiträgt, welche kognitiven Prozesse dabei eine Rolle spielen und welche Fehlkonzepte entstehen. Die Autorinnen schließen eine Forschungslücke, da sie situationsorientiert unter Heranziehung einer authentischen Situation testen und zudem Prozessdaten (Think-aloud Audiografien) zur Erklärung der Produktdaten (Testergebnisse) heranziehen. Die Diskussion von Rudeloff (Abschnitt 5) würdigt die in den Beiträgen dargestellten Ansätze und gibt einen Ausblick auf zukünftige Forschung.

\section{Messung von Finanzkompetenz}

In der Forschung zur Financial Literacy kann zwar auf einen Bestand an existierenden Instrumenten zur Messung finanzieller Kompetenzen zurückgegriffen werden, welche aber auf Grund unterschiedlichster Schwerpunktsetzung hinsichtlich einer ganzheitlichen Kompetenzfeststellung ausbaufähig erscheinen. Differenziert nach internationalen, vorwiegend US-amerikanischen, und nationalen Instrumenten zur Erfassung von Financial Literacy soll hierzu im Folgenden ein kurzer Überblick gegeben werden, bevor Desiderata für zukünftige Testverfahren diskutiert werden. Der vorliegende Beitrag erhebt nicht den Anspruch, ein vollumfassendes Review aller bestehenden Instrumente zum Assessment von Financial Literacy zu liefern. Vielmehr wird der Fokus auf 
internationale wie nationale Instrumente aus der jüngeren Vergangenheit gelegt, die im Bereich des Schul- bzw. Bildungskontextes für junge Lernende angelegt sind und die exemplarisch den Stand der Instrumentenentwicklung verdeutlichen sowie die Weiterentwicklungen innerhalb des Symposiums betonen sollen. ${ }^{2}$

Die Herausforderungen bei der Erfassung von Financial Literacy bzw. Finanzkompetenz als latente Konstrukte liegen bereits in der Definition der Begriffe und in ihrer nationalen wie internationalen Anschlussfähigkeit begründet (s. Fußnote 1). In Anlehnung an Weinert $(2001,2002)$ werden im Folgenden auch Messverfahren exemplarisch betrachtet, die über eine Betrachtung rein kognitiver Facetten im Assessment hinausgehen und auch emotional-motivationale, volitionale und soziale Facetten berücksichtigen. Dies soll im Folgenden und ebenfalls in der angefügten tabellarischen Übersicht der Assessment-Tools näher dargestellt werden. Weiterhin gibt die angefügte Tabelle (s. Appendix) zum besseren Verständnis der Messung Aufschluss über die dimensionale Struktur der Messmodelle sowie die Art der Messverfahren.

Insbesondere in den USA haben die Entwicklung und der Einsatz von standardisierten und normierten Testinstrumenten zur Messung finanzieller Kompetenzen oder deren Facetten eine lange Tradition und waren auch lange Zeit verbreiteter als in Deutschland (Hausmann \& Retzmann 2014). Als Pionierarbeit können hierfür die vom amerikanischen Council of Economic Education (CEE) entwickelten Testbatterien genannt werden. Diese stellen auf die ebenfalls vom CEE entwickelten National Standards for Financial Literacy $a b$, die insbesondere für den Bereich persönlicher Finanzfragen sechs Inhaltsbereiche (Einkommen, Kauf von Gütern und Dienstleistungen, Sparen, Kreditnutzung, finanzielle Investitionen, Versicherungen) beschreiben und für eine fundierte Finanzbildung eines Individuums als essenziell angesehen werden (Walstad \& Rebeck 2016a; CEE 2013). Entwickelt wurden der Basic Finance Test (BFT) für die oberen Stufen der Grundschule (upper elementary) und die Einstiegsklassen der Mittelschule (lower middle school) (Walstad \& Rebeck 2016a), der Test of Financial Knowledge (TFK) für die obere Mittelstufe (upper middle school) und die Sekundarstufe I (lower high school) (Walstad \& Rebeck 2016b) sowie der Test of Financial Literacy (TFL) für die Sekundarstufe II und die Abschlussklassen (high school) (Walstad \& Rebeck 2016c). In all diesen Tests werden finanzielles Wissen und Verständnis mittels Single-Choice-Antwortformaten mit kurzen Item-Stämmen erfasst.

Während die vom CEE entwickelten Instrumente mit 35 (BFT), 40 (TFK) oder 45 Items (TFL) eine große Breite an finanziellen Inhaltsbereichen detailliert abbilden, verfolgen Lusardi und Mitchell (2011) einen anderen Ansatz.

2 Für weitere Übersichten siehe u. a. Schürkmann und Schuhen (2013) sowie Geiger, Meretz und Liening (2016). 
Basierend auf den Grundprinzipien Einfachheit, Relevanz, Kürze und Fähigkeit zur Differenzierung operationalisieren sie einen Indikator für Finanzwissen anhand nur weniger Items im Single-Choice-Format (Lusardi \& Mitchell 2011). Die Autoren differenzieren dabei zwischen Fragen zu basalen Finanzkonzepten (basic) und Fragen zu anspruchsvolleren Finanzmechanismen (sophisticated). Erstere adressieren u. a. die Rechenfähigkeit, Fachkenntnisse zu den Themenbereichen Inflation oder Zinseszinsen sowie das Wissen zu Risiken beim Aktienkauf in drei bis fünf Single-Choice-Items, während letztere weitergehende finanzielle Sachverhalte, wie z. B. Funktionsweisen von Aktienmärkten oder Volatilität, in acht Single-Choice-Items erfassen (Lusardi \& Mitchell 2009).

Im Kontext großangelegter internationaler Schulleistungsstudien wurde die Thematik der Financial Literacy erstmals 2012 auch in das Programme for International Student Assessment (PISA) aufgenommen und anhand von 40 Fragen aus den Inhaltsbereichen Geld und Transaktionen, Finanzplanung, Risiko und Belohnung sowie Finanzwelt (financial landscape) mittels Pen-andPaper-Test erfasst (OECD 2013). Die Aufgaben stellen dabei verstärkt einen Bezug zur lebensweltlichen Realität von 15-jährigen Schüler*innen her, indem mit (kurzen) Fallbeispielen Lebensereignisse oder -umstände, wie der Kauf einer Motorroller-Versicherung, aufgegriffen werden. Das Antwortformat variiert dabei zwischen auszuformulierenden Kurzantworten (constructed responses), kleineren Berechnungen sowie Auswahl- oder Ankreuzaufgaben (selected responses). Neben der Betrachtung dieser überwiegend kognitiven Facetten der Financial Literacy wurden im Nachgang an die Erhebung auch SchülerFragebögen ausgegeben, die nach nicht-kognitiven Aspekten, wie beispielsweise dem eigenen Sparverhalten oder dem Grad des Zugangs zu Geldinformationen und Finanzprodukten, fragten, um so Rückschlüsse auf das Interesse und die Erfahrung der Schüler*innen hinsichtlich Finanzfragen zu ermöglichen (OECD 2013).

Im deutschsprachigen Raum wurden in den vergangenen Jahren ebenso verstärkt Bemühungen zur Erforschung finanzieller Bildung unternommen. Hier wurden verschiedene Wege gewählt: Zum einen wurden die erwähnten Instrumente des TFL (Förster, Happ \& Molerov 2017) oder des TFK (Kraitzek, in Vorb.) für den Einsatz im deutschsprachigen Raum adaptiert und validiert. Des Weiteren wurden neue objektive Messverfahren entwickelt, welche einige der Limitationen der US-amerikanischen Instrumente überwinden. Hierzu zählen u. a. die von Rudeloff (2019) und Schürkmann (2017) entwickelten Testverfahren, bei denen die Aufgaben stärker situativ eingebunden sind. Parallel dazu gab es Anstrengungen, auch nicht-kognitive oder metakognitive Facetten der Finanzkompetenzen separat $\mathrm{zu}$ erfassen. So adaptierte beispielsweise Barry (2014) verschiedene englischsprachige Fragebögen zur Einstellung zu Geld ins Deutsche und fasste diese zu fünf neuen Rating-Skalen zusammen. Mit diesem Instrument wird u. a. abgefragt, welche subjektive Bedeutsamkeit 
ein Individuum der Geldthematik zuschreibt oder ob individuelle Macht- und Geltungsbedürfnisse durch den Besitz und den Umgang mit Geld erfüllt werden. Die von Barry entwickelten Skalen wurden u. a. auch in den Studien von Schürkmann (2017), Grohs-Müller (2018) und Rudeloff (2019) verwendet, um Einstellungen zu Geld zu erfassen, wobei das Instrument bei Grohs-Müller in einer modifizierten Version erweitert um die Faktoren „Glück durch Geld“ und „Angst und Sorgen“"(Grohs-Müller 2018, 91) zum Einsatz kommt. Bender und Barry (2013) gingen einen ähnlichen Weg, indem sie den von Breuer (1999) ins Deutsche adaptierten Trait Self Regulation Questionnaire (TSRQ) zur Erfassung der Selbstregulationsfähigkeit bei finanziellen Handlungen weiterentwickelten. Darüber hinaus wurden von Rudeloff (2019) und Grohs-Müller (2018) Bemühungen unternommen, die finanzielle Sozialisation zu erfassen. Rudeloff (2019) bezog zudem die Selbstwirksamkeit im Umgang mit Finanzen mit einer Adaption nach Jerusalem und Schwarzer (1999) in die Untersuchung ein, während Grohs-Müller (2018) noch Rating-Skalen für selbstberichtete Verhaltensweisen im Umgang mit Geld entwickelte.

Der Ansatz, nicht nur Wissen, sondern auch dessen Anwendung sowie nicht-kognitive Dispositionen, z. B. Einstellungen, Motivation oder Wertvorstellungen zu berücksichtigen, ist generell kompatibel mit einem ganzheitlichen Kompetenzverständnis, so wie es z. B. auch Weinert (2001) definiert hat (s. o.). Sofern allerdings nicht-kognitive Konstrukte in Studien überhaupt Berücksichtigung finden, erfolgt dies in der Regel in Form separat erfasster Rating-Skalen, welche einzelne Facetten, wie z. B. die Einstellung zu Geld, erfassen. Dies hat zur Folge, dass dann in der Regel kein Bezug auf einen bestimmten Inhaltsbereich finanzieller Kompetenz (z. B. Sparen, Versichern) genommen und der situative Kontext der vor- oder nachgelagerten kognitiven Kompetenzmessung nicht berücksichtigt wird. Neue Verfahren müssen kognitive und nicht-kognitive Facetten möglichst simultan im gleichen Szenario und der gleichen Situation erfassen, da dies die Validität der Konstrukte deutlich erhöhen und bessere Rückschlüsse auf deren Zusammenhänge bei der Lösung von Aufgaben ermöglichen würde.

Weiterhin herrscht bislang eine starke, wenn nicht sogar ausnahmslose Fokussierung auf die durch Tests gemessenen Kompetenzen am Ende einer Intervention oder Lerneinheit, während der eigentliche Prozess der Problemlösung in seiner Feinheit bisher kaum betrachtet wird. Aber gerade die Prozessperspektive wäre aus diagnostischer Sicht von hoher Relevanz, um Aussagen treffen zu können, welche kognitiven und nicht-kognitiven Prozesse eine Rolle spielen und in welchen Phasen des Problemlösungs- oder Entscheidungsprozesses die Proband*innen bestimmte Fehler oder Fehlkonzeptionen entwickeln.

An diesen Forschungsdesiderata setzten die folgenden Beiträge des Symposiums an. Wuttke, Siegfried und Aprea setzen erstmals einen SJT zur Erfassung von Finanzkompetenz ein. Hommel und Fürstenau untersuchen, wie sich 
die finanziellen Kompetenzen von Studierenden entwickeln, die informelle Lerngelegenheiten zur Finanzthematik im Internet wahrnehmen. Dies geschieht im konkreten Fall anhand des komplexen Finanzproduktes der Baufinanzierungsdarlehen.

\section{Messung von Financial Literacy mit einem Situational Judgement Test - sind bestimmte Personengruppen wirklich schlechter oder liegt es am Messinstrument?}

Problemstellung und konzeptuelle Grundlagen

Aktuelle Studien zu Financial Literacy zeigen u. a., dass männliche Probanden in Tests oft besser abschneiden als weibliche (z. B. Bucher-Koehnen et al. 2017), und dass Personen mit Migrationshintergrund und solche mit niedrigem Bildungsniveau oft schlechter abschneiden als solche ohne Migrationshintergrund (z. B. Happ \& Förster 2019) und/oder hohes Bildungsniveau (z. B. Lusardi \& Mitchell 2014). Diese Befunde werden häufig als Indiz für die Schwäche und den besonderen Förderbedarf der genannten Gruppen angesehen. Wie Forschungsarbeiten aus angrenzenden Gebieten wie etwa der ökonomischen Bildung (z. B. Siegfried \& Wuttke 2019) oder der Mathematik (z. B. Lindner et al. 2015; Reardon et al. 2018) sowie zahlreichen anderen Assessmentbereichen (z. B. Saygin 2019) zeigen, können solche Gruppenunterschiede jedoch (zumindest partiell) auch aus Benachteiligungen resultieren, die auf das Messinstrument zurückzuführen sind. Eine systematische Untersuchung dieser Interpretation liegt im Bereich von Financial Literacy bislang noch nicht vor, ist jedoch von zentraler Bedeutung sowohl für die wissenschaftliche Erklärung von Leistungsunterschieden als insbesondere auch für die praktische Entscheidungsfindung in Bezug auf entsprechende Bildungsmaßnahmen. Die im Folgenden vorgestellte Studie möchte einen Beitrag zur Schließung dieser Forschungslücke leisten, indem ein alternativer Zugang zur Messung von Financial Literacy vorgestellt und eruiert wird, ob und inwieweit dieser Zugang zu Abweichungen von bisherigen Ergebnissen führt. Die Studie ist Bestandteil eines breit angelegten Forschungsprogramms zur Entwicklung eines validen Assessments von Financial Literacy.

Der alternative Zugang basiert in Anlehnung an Ansätze aus der Wirtschaftsdidaktik auf einer kompetenzorientierten Konzeptualisierung von Financial Literacy (Aprea \& Wuttke, 2016; Wuttke \& Aprea, 2018). Zur Entwicklung entsprechender Kompetenzfacetten wurden zunächst Interviews mit 40 internationalen Experten aus dem Bildungs- und Finanzbereich geführt (Aprea et al. 2015). Es wurden 9 Facetten ermittelt, für die Testitems gemäß 
dem Ansatz des SJT (McDaniel et al. 2007) konstruiert wurden. Dieser Ansatz wurde gewählt, da die Messung von Financial Literacy im Sinne eines ganzheitlichen Konstruktes einen Messzugang erfordert, der in der Lage ist, komplexe Fähigkeiten zu erfassen und diese durch ein Set an Aufgaben beobachtbar zu machen, deren Anforderungen möglichst nahe an der erwarteten Performanz liegen. Solche SJT präsentieren realistische, hypothetische Situationen, zu denen sich die Probanden ,angemessen“ verhalten müssen. Bisherige Befunde zeigen, dass mit dieser Art der Testung Verhalten in Echtsituationen gut vorhergesagt werden kann (Kahmann 2014).

\section{Forschungsfragen}

Die Frage, die sich v. a. mit Blick auf die neue Testkonzeption stellt, ist, ob sich in den verschiedenen Gruppen die Ausprägung von Financial Literacy unterscheidet (wie es bisherige Befunde nahelegen), oder ob von Unterschieden auf Basis des eingesetzten Instruments, also einem Testbias auszugehen ist. Deshalb wird in einem ersten Schritt geprüft, ob ein latenter Mittelwertvergleich der Gruppen (weibliche vs. männliche Testpersonen, Personen mit oder ohne Migrationshintergrund und Personen mit akademischen vs. beruflichem Bildungshintergrund) zulässig ist. Hierfür ist zunächst zu prüfen, ob für die zu untersuchenden Gruppen Messinvarianz angenommen werden kann. Sollte das der Fall sein, werden Gruppenunterschiede geprüft.

\section{Untersuchungsdesign}

Es wurden insgesamt 22 Items zu den Kompetenzfacetten „Kontrolle der eigenen finanziellen Situation“, „Budgetierung“ und „Umgang mit Geld“ eingesetzt. Die Skalen haben zufriedenstellende Reliabilitäten (Kontrolle der eigenen finanziellen Situation, 9 Items, max. 36 Punkte, $\alpha=.754$; Budgetierung, 6 Items, max. 24 Punkte, $\alpha=.573$; Umgang mit Geld 7 Items, max. 28 Punkte, $\alpha=.691$ ). An der Erhebung nahmen 206 junge Erwachsene teil (weiblich 52,2\%; Altersdurchschnitt 20,06; Bildungshintergrund: 105 Studierende, 101 berufliche Ausbildung (Vollzeitschulen und duale Ausbildung), 62\% mit Migrationshintergrund $=$ Muttersprache beider Eltern ist nicht Deutsch).

Die Daten wurden mit Blick auf das Geschlecht, den Bildungshintergrund und den Migrationshintergrund Mittelwertvergleichen unterzogen. Vor Überprüfung von Mittelwertunterschieden werden folgenden Messinvarianzmodelle (mit zunehmender Restriktion) geprüft: Konfigurale Messinvarianz, metrische Invarianz, skalare Invarianz, strikte Invarianz/Invarianz der Messfehler (Temme \& Hildebrand 2008). 
Ergebnisse

I Geschlecht: Im Rahmen der konfiguralen Invarianzmodelle zu den drei Facetten von Financial Literacy wurden alle Faktorladungen, Intercepts und Residualvarianzen über beide Geschlechter hinweg frei gesch tzt. Die Fit-Statistiken zeigen zunächst, dass der Modelfit bei allen drei konfiguralen Invarianzmodellen angenommen werden kann (,Kontrolle“: $\chi 2=61.07, \mathrm{p}=.098, \mathrm{df}=$ $48, \mathrm{CFI}=.95 ; \mathrm{RMSEA}=.037$, Pclose $=.801 ;$,Budgetierung““ $: \chi^{2}=41.103, \mathrm{p}$ $=.008, \mathrm{df}=22, \mathrm{CFI}=.94 ; \mathrm{RMSEA}=.066$, Pclose $=.185 ;$, Umgang mit Geld“ $\chi 2=36.555, \mathrm{p}=.006, \mathrm{df}=18, \mathrm{CFI}=.89 ; \mathrm{RMSEA}=.072$, Pclose $=.131$ ), auch wenn der CFI für die Facette „Umgang mit Geld“ zu niedrig ausfällt. Damit kann angenommen werden, dass die drei Faktoren in beiden Gruppen in hnlicher Weise konzeptualisiert sind. Hinsichtlich der Gleichheitsrestriktionen auf den Faktorladungen, die im Rahmen der metrischen Invarianz gesetzt wurden, verhält es sich allerdings je nach Facette unterschiedlich. Während sich für die Facette „Kontrolle der eigenen finanziellen Situation“ und „Budgetierung“ die Modellpassung nicht bedeutsam verschlechtert $(\triangle \mathrm{CFI} \leq$. |.02|, $\triangle$ RMSEA $\leq$ .015) und somit abgeleitet werden kann, dass die Maßeinheit der beiden Skalen für weibliche und männliche Testteilnehmer identisch ist, stellt sich für die Facette „Umgang mit Geld“ bei dieser Modellrestriktion eine sich weiter verschlechternde Modellpassung heraus $(\chi 2=47.516, \mathrm{p}=.003, \mathrm{df}=24, \mathrm{CFI}=$ .86 ; RMSEA $=.07$, Pclose $=.122$ ). Erst durch die Freisetzung der Faktorladung eines Items (dies fand auf Basis der Größe des Modifikationsindexes statt) kann ein ähnlicher Modelfit wie bei der konfiguralen Messinvarianz erreicht werden $(\chi 2=43.184, \mathrm{p}=.007, \mathrm{df}=23, \mathrm{CFI}=.89$; RMSEA $=.066$, Pclose $=.175)$.

Für die Prüfung der starken/skalaren Messinvarianz wurden neben den Faktorladungen auch die Intercepts der einzelnen für die latenten Skalen herangezogenen Items für männliche und weibliche Probanden gleichgesetzt. Hierbei wird durch die Verschlechterung der Modellpassungen $(\triangle \mathrm{CFI}>.|.02|)$ deutlich, dass für keine der Facetten diese Modellrestriktion angenommen werden kann. Auch hier lohnt es sich allerdings zu untersuchen, ob die starke Invarianz zumindest partiell erreicht werden kann. Hierfür wurden für die Facette „Kontrolle der eigenen finanziellen Situation“, „Budgetierung“ und „Umgang mit Geld“ " je ein Intercept freigesetzt, während die anderen Faktorladungen auf Gleichheit über die beiden angenommenen Subgruppen restringiert wurden. Die Teststatistiken des Modellvergleichs zeigen, dass durch diesen Zugang partielle Messinvarianz für die beiden Facetten ,Kontrolle der eigenen finanziellen Situation“ $(\chi 2=86.701, \mathrm{p}=.002, \mathrm{df}=65, \mathrm{CFI}=.91$; RMSEA $=.041$, Pclose $=.736)$ und "Budgetierung" $(\chi 2=59.521, \mathrm{p}=.006, \mathrm{df}=35, \mathrm{CFI}=.92$; RMSEA $=.059$, Pclose $=.259)$ erreicht werden konnte. Für die Facette „Umgang mit Geld" ist die Modellpassung allerdings nicht gegeben $(\chi 2=52.835$, $\mathrm{p}=.002, \mathrm{df}=27, \mathrm{CFI}=.85$; RMSEA $=.069$, Pclose $=.128)$. Für die Facette 
„Budgetierung“ zeigt sich darüber hinaus, dass sogar eine strikte Messinvarianz angenommen werden kann $(\chi 2=70.489, \mathrm{p}=.004$, df $=42, \mathrm{CFI}=.91$; RMSEA $=.058$, Pclose $=.265$ ).

Vor diesem Hintergrund ist lediglich für die Facetten „Kontrolle der eigenen finanziellen Situation“ und „Budgetierung“ die Voraussetzung für einen sinnvollen Vergleich der Mittelwerte dieser Skalen gegeben. Der T-Test zeigt in diesem Zusammenhang lediglich einen signifikanten Unterschied für die Facette „Kontrolle der eigenen finanziellen Situation“ zum Vorteil weiblicher Probanden (Kontrolle: $\mathrm{t}(182)=-3.058, \mathrm{p}=.003$; Budgetierung: $\mathrm{t}(186)=-$ $1.018, \mathrm{p}=.310)$.

Aus Platzgründen kann an dieser Stelle nicht ausführlicher auf die Analyse der Messinvarianz zu Bildungshintergrund und Migrationshintergrund eingegangen werden. Es wurde so vorgegangen, wie bei der Analyse der Messinvarianz bei männlichen/weiblichen Probanden (ausführlich Wuttke, Siegfried \& Aprea, in Vorbereitung). Zusammenfassend lässt sich festhalten:

II Bildungshintergrund: Es kann eine (partielle) starke Messinvarianz für die beiden Facetten „Kontrolle der eigenen finanziellen Situation“ und „Budgetierung“ erreicht werden. Für diese Facetten ist der Vergleich der Mittelwerte der Skalen zwischen Testpersonen mit beruflichem vs. akademischem Bildungshintergrund zulässig. T-Tests zeigen, dass Probanden, die aktuell ein Studium absolvieren in der Facette „Budgetierung“ signifikant besser abschneiden als diejenigen, die im beruflichen Schulwesen sind (Kontrolle: $\mathrm{t}(184)=-0.491, \mathrm{p}=.62$; Budgetierung: $\mathrm{t}(187)=-3.073, \mathrm{p}<.01)$.

III Migrationshintergrund: Für alle drei Facetten ist die Voraussetzung für die Testung von Mittelwertunterschieden zwischen den Subgruppen gegeben. Die Ergebnisse der T-Tests verweisen darauf, dass für die Facetten „Kontrolle der eigenen finanziellen Situation“ und „Budgetierung“ keine signifikanten Gruppenunterschiede bezüglich des Migrationshintergrundes vorliegen (Kontrolle: $\mathrm{t}(181)=-1.367, \mathrm{p}=.17$; Budgetierung: $\mathrm{t}(184)=0.817, \mathrm{p}=.42$ ), allerdings ist dies der Fall für die Facette „Umgang mit Geld“ $(\mathrm{t}(179)=-1.955$, $\mathrm{p}=.05)$ und zwar zum Vorteil für diejenigen Probanden, die keinen Migrationshintergrund aufweisen.

\section{Relevante Implikationen und Limitationen}

Die Analyse der Messinvarianz zeigt, dass der entwickelte Test für die Facetten Kontrolle der eigenen finanziellen Situation und Budget erstellen bei allen betrachteten Gruppen Messinvarianz aufweist, Gruppenvergleiche sind damit möglich und potentielle Unterschiede sind nicht auf einen Testbias zurückzuführen. Für die Facette Umgang mit Geld kann keine Messinvarianz angenommen werden. 
Entgegen der Befunde aus vielen bisherigen Studien zeigen sich bei der Analyse der Mittelwertunterschiede keine systematischen Nachteile für bestimmte Gruppen: Mit Blick auf das Geschlecht zeigt sich, dass weibliche Probanden bei der Budgetierung dieselbe Leistung zeigen wie männliche Probanden, bei der Facette Kontrolle der eigenen finanziellen Situation schneiden sie sogar signifikant besser ab. Mit Blick auf den Bildungshintergrund zeigt sich bei der Facette Budgetierung ein erwartungskonformer Vorteil für Personen mit akademischem Hintergrund, bei den übrigen Facetten sind keine Unterschiede zu finden.

Insgesamt legen die Ergebnisse damit den Schluss nahe, dass häufig die Art der Testung mit möglichen Vor- oder Nachteilen für bestimmte Gruppen verbunden ist (s. a. Greimel-Fuhrmann \& Silgoner 2018; Siegfried \& Wuttke 2019). Dies scheint im vorliegenden SJT weniger der Fall zu sein.

Die Facette Umgang mit Geld ist vor dem Hintergrund der fehlenden Messinvarianz zu überarbeiten. Hierzu ist eine Studie mit Nutzung lauten Denkens geplant, die Hinweise geben soll, wie die Items der Facette zu überarbeiten sind.

\section{Kognitive Prozesse und Fehlkonzepte beim informellen Erwerb von Finanzkompetenz über Baufinanzierung}

\section{Relevanz und Zielsetzung}

Mit dem Ziel, die Finanzkompetenz von Individuen zu fördern, haben - insbesondere seit der Finanzkrise im Jahr 2007/2008 - viele Länder entsprechende Bildungsprogramme zur Financial Education entwickelt und zum Teil curricular verankert. Dadurch sollen negative Auswirkungen potenzieller Fehlentscheidungen sowohl auf persönliche Finanzsituationen (OECD 2013) als auch auf ganze Volkswirtschaften vermieden werden. Neben diesen Bildungsprogrammen, die als formales Lernen gewertet werden können, ist es jedoch auch möglich, Finanzkompetenz informell zu erwerben. Informeller Erwerb von Finanzkompetenz stand jedoch bislang nicht im Zentrum von Forschungsbemühungen in diesem Kontext.

Insbesondere das Internet bietet eine Fülle von Informationen, die Individuen bedarfsorientiert nutzen können, um sich in einer bestimmten Domäne, z. B. bezogen auf komplexe Finanzprodukte, informell weiterzubilden (Dennen \& Wang 2002; Werquin 2010). Dieses informelle online Lernen ist insgesamt nicht so gut erforscht wie informelles Lernen im Allgemeinen. Bezogen auf die Frage, welche Designprinzipien informelles online Lernen von Erwachsenen unterstützen, zeigt eine neuere Metasynthese, dass es insbesondere 
auf Möglichkeiten zur Interaktion (Wissen und Erfahrungen zu Themen mitteilen) sowie auf gut gegliederte und markierte sowie klar betitelte Informationen ankommt. Weitere Prinzipien, wie eine möglichst umfangreiche Informationsbasis oder hohe soziale Präsenz, sind noch nicht hinreichend gesichert (Holland 2019). Auf Seiten des Individuums spielen Faktoren, wie die Fähigkeit selbstgesteuert zu lernen, vor allem aber Vorwissen und Vorerfahrung, eine Rolle (Maier et al. 2014; Cerasoli et al. 2018). Die Forschung zeigt, dass informelles online Lernen nicht immer erfolgreich ist. Es können beispielsweise Fehlkonzepte als ,inkorrektes Wissen“ (Eitel, Dersch \& Renkl 2019, 454) entstehen. Aus Verständnisproblemen und Unsicherheiten, die nicht geklärt werden, entwickeln sich solche Fehlkonzepte. Diese sind problematisch, da sich aus ihnen handlungsrelevante (aber eben fehlerhafte) Überzeugungen entwickeln können (ebd., 453).

Im Rahmen einer Studie wurde geprüft, inwiefern online verfügbare Informationen das informelle Lernen unterstützen, welche kognitiven Aktivitäten dabei eine Rolle spielen und welche Verständnisschwierigkeiten entstehen können. Konkret stand ein komplexes Finanzprodukt, in diesem Fall Baufinanzierungsdarlehen, im Zentrum des Interesses. Mit Blick auf die Messung von Finanzkompetenz greifen wir gezielt den Aspekt der Situationsbezogenheit auf, da sich die Probanden in eine komplexe Entscheidungssituation über die Aufnahme eines Baufinanzierungsdarlehens hineinversetzen und sich aus diesem Kontext heraus Wissen aneignen müssen. Weiterhin beziehen wir in die Messung sowohl Produktdaten (Testergebnisse) als auch Prozessdaten (Thinkaloud-Protokolle, die Fehlkonzepte verdeutlichen) ein, um dadurch Ergebnisse besser erklären und Anhaltspunkte für instruktionale Unterstützung gewinnen zu können.

\section{Forschungsfragen}

I. Tragen Webseiten von Banken im Rahmen informellen Lernens dazu bei, die Entwicklung von Finanzkompetenz potentieller Darlehensnehmer*innen in Bezug auf Baufinanzierungsdarlehen zu fördern?

II. Welche kognitiven Aktivitäten sind mit der Exploration von Webseiten zu Baufinanzierungen durch potentielle Darlehensnehmer*innen verbunden?

III. Welche Verständnisschwierigkeiten haben potentielle Darlehensnehmer*innen beim Explorieren eines Darlehenskalkulators? 


\section{Methode}

Die Stichprobe umfasst 45 Studierende der Wirtschaftswissenschaften (Tab. 1), die zufällig einer Experimentalgruppe (EG) oder einer Kontrollgruppe (KG) zugewiesen wurden.

Tab. 1: Stichprobe

\begin{tabular}{lccccc}
\hline & $\mathrm{n}$ & $\%$ & Alter M & Geschlecht & Think-aloud \\
\hline EG & 23 & 51.1 & 21.8 & $6 \mathrm{~m}, 17 \mathrm{w}$ & 12 \\
KG & 22 & 48.9 & 22.5 & $4 \mathrm{~m}, 16 \mathrm{w}, 2 \mathrm{~d}$ & 6 \\
Total & 45 & 100 & 22.2 & $10 \mathrm{~m}, 33 \mathrm{w}, 2 \mathrm{~d}$ & 18 \\
\hline
\end{tabular}

Quelle: eigene Abbildung

Die Proband*innen der EG explorierten die Webseite der Commerzbank zum Darlehenskalkulator (Hommel et al. 2017; Fürstenau \& Hommel 2018). Dieser Kalkulator wurde ausgewählt, da er mehr Informationen und somit potenziell mehr Lerngelegenheiten zur Verfügung stellt als die Kalkulatoren anderer Banken. Die Qualität der Informationen dieses Kalkulators ist vergleichbar mit denen der Kalkulatoren anderer Banken (Fürstenau et al. 2016). Die Proband*innen der KG hatten keinen Zugang zu (Internet-)Informationen über Baufinanzierungen. Im Rahmen einer Fallsituation mussten die Proband*innen beider Gruppen darüber entscheiden, ob sie unter den gegebenen Bedingungen ein Baufinanzierungsdarlehen aufnehmen. Die Entscheidung war zu begründen.

Vor der Exploration bearbeiteten alle Teilnehmenden einen Pretest zu numerischen Fähigkeiten (Prozent-, Zins- und Annuitätenrechnung) sowie eine Selbsteinschätzung in Bezug auf Finanzwissen zu Baufinanzierungsdarlehen. Insgesamt waren im Pretest sieben Punkte erreichbar. Nach der Exploration absolvierten alle Personen einen Posttest mit vier offenen Items, u. a. zu den Komponenten einer Annuität, dem Konzept Zinsfestschreibung etc., sowie neun Einfachwahlaufgaben zum Inhaltsbereich Baufinanzierung. Im Posttest waren maximal 21 Punkte erreichbar. Beispiele zu den Messinstrumenten finden sich bei Hommel et al. (2017) sowie Fürstenau und Hommel (2019).

Während der Exploration und bei der Bearbeitung der Fallsituation externalisierten 12 Proband*innen der EG und sechs Proband*innen der KG ihr Denken laut (Fürstenau \& Hommel 2019). Die Think-aloud-Daten wurden audiografiert, transkribiert und mithilfe einer kategorialen Inhaltsanalyse ausgewertet (Krippendorf 2012). Dazu wurde das Kategoriensystems für ,self-explanations' von Chi et al. (1989) adaptiert. Der Kodierleitfaden wurde von 
zwei Kodierenden gemeinsam entwickelt. In diesem Prozess stand die semantische Gültigkeit im Fokus, um die Bedeutung der kodierten Aussagen beizubehalten bzw. zu rekonstruieren (Mayring 2015). Die Aussagen wurden in den Hauptkategorien (1) Erklären/Elaborieren (Äußerungen zu Informationen/Fakten, denen Bedeutung zugewiesen wird, die evaluiert werden, oder zu Konsequenzen, die durchdacht werden), (2) Monitoring (Aussagen zum eigenen Lernen, wie zum Verstehen, zu Unsicherheiten im Verständnis oder zum Nichtverstehen) sowie (3) Andere (Wahrnehmen, Paraphrasieren von Informationen; Fragen zum Inhalt, metastrategische Aussagen) zugeordnet.

\section{Ergebnisse}

In den Ergebnissen des Pretests (Tab. 2) unterschieden sich die Proband*innen der $\mathrm{EG}$ und $\mathrm{KG}$ nicht signifikant $(\mathrm{n}=45, \alpha=.05, \mathrm{t}=-1.071, \mathrm{df}=43, \mathrm{p}=.290$, $\mathrm{d}_{\text {Cohen }}=0.315$, Teststärke $\left.(1-\beta)=.26\right)$. Auch im Posttest erreichten die Gruppenunterschiede nicht das Signifikanzniveau $\left(\mathrm{t}=-1.180, \mathrm{df}=43, \mathrm{p}=.245, \mathrm{~d}_{\mathrm{Co}}\right.$ hen $=0.355,(1-\beta)=.31)$. Mithilfe einer mixed ANOVA wurden der Wissensstand zu den beiden Zeitpunkten (Innersubjektfaktor) und der Zwischensubjektfaktor Gruppe gemeinsam analysiert. Allerdings zeigt sich in unserem Fall ausschließlich ein signifikanter Einfluss des Faktors Zeit $(F(1,43)=241.28$, $\mathrm{p}<.001$, partielles $\eta^{2}=.85$.) und kein Interaktionseffekt zwischen der Zeit und der Gruppe $\left(F(1,43)=0.923, p>.05\right.$, partielles $\left.\eta^{2}=.021\right)$. Insofern trägt informelles Lernen unter Nutzung von Webseiten scheinbar nicht zum Erwerb von Finanzkompetenz bei (Forschungsfrage I).

Tab. 2: Punkte im Pretest und Posttest

\begin{tabular}{ccccccccc}
\hline & \multicolumn{4}{c}{ KG } & \multicolumn{5}{c}{ EG } \\
& M & SD & Min & Max & M & SD & Min & Max \\
\hline Pretest & 3.00 & 1.45 & 0 & 6 & 3.43 & 1.27 & 1 & 6 \\
Posttest & 11.18 & 3.74 & 3 & 17 & 12.70 & 4.78 & 4 & 21 \\
\hline
\end{tabular}

Quelle: eigene Abbildung 
Um Anhaltspunkte dafür zu gewinnen, warum dies der Fall ist, wurden die kognitiven Prozesse analysiert (Forschungsfrage II) und die Think-aloud-Daten der Proband*innen der EG denen der KG gegenübergestellt (Tab. 3).

\section{Tab. 3: Kategorien der Think-aloud-Kodierung}

Think-aloud-Kategorie

(1) Erklären/Elaborieren

\begin{tabular}{cccc}
\multicolumn{2}{c}{ KG } & \multicolumn{2}{c}{ EG } \\
\hline $\mathrm{n}$ & $\%$ & $\mathrm{n}$ & $\%$ \\
\hline 236 & 49.2 & 551 & 34.1
\end{tabular}

Evaluieren von Fakten, Ergebnissen, Informationen

$\begin{array}{llll}30 & 6.3 & 133 & 8.2\end{array}$

Erklären oder Ableiten von Konsequenzen

$\begin{array}{llll}188 & 39.2 & 289 & 17.9\end{array}$

Zuweisen von Bedeutungen zu Aussagen

Definieren, Verfeinern, Filtern oder Erweitern von Bedingungen, die Entscheidung beeinflussen

\begin{tabular}{lcccc}
\hline (2) Monitoring & 47 & 9.8 & 112 & 6.9 \\
Aussagen über Nichtverstehen & 33 & 6.9 & 58 & 3.6 \\
Aussagen zu Unsicherheiten im Verstehen & 9 & 1.9 & 28 & 1.7 \\
Aussagen zum Verstehen von Informationen & 5 & 1.0 & 26 & 1.6 \\
\hline (3) Andere & 197 & 41 & 952 & 59 \\
Auswählen aus einem Drop-down-Menü & 18 & 3.8 & 136 & 8.4 \\
Metastrategische Aussagen & 35 & 7.3 & 217 & 13.4 \\
Paraphrasieren gegebener Informationen & 29 & 6.0 & 118 & 7.3 \\
Wahrnehmen oder Erinnern von Informationen & 11 & 2.3 & 93 & 5.8 \\
Fragen zum Inhalt & 16 & 3.3 & 157 & 9.7 \\
Lesen von Informationen & 88 & 18.3 & 231 & 14.3 \\
\hline Total & 480 & 100 & 1615 & 100 \\
\hline
\end{tabular}

Quelle: eigene Abbildung

Im Vergleich der Häufigkeiten der Think-aloud-Kodierungen wird deutlich, dass der relative Anteil von Aussagen in der Kategorie Erklären/Elaborieren mit 49.2\% in der Kontrollgruppe (KG) über dem der Experimentalgruppe (EG) mit 34.1\% der jeweils kodierten Aussagen liegt. Innerhalb dieser Kategorie entfallen $39.2 \%$ in der KG und nur $17.9 \%$ in der EG auf das Erklären/Ableiten 
von Konsequenzen. Auch in der Kategorie Monitoring liegt der relative Anteil der Aussagen in der KG mit 9.8\% über dem der EG mit 6.9\%. Dagegen zeigt sich der überwiegende Anteil der kodierten Aussagen in der EG mit 59\% in der Kategorie Andere im Vergleich zur KG mit 41.0\%. In der EG beziehen sich diese Aussagen mit 8.4\% auf das Auswählen von Informationen, z. B. aus Drop-down-Menüs des Kalkulators, und mit 13.4\% auf metastrategische Aspekte, wie das Reflektieren bereits vollzogener Schritte, deren Reihenfolge oder noch auszuführende Aktionen. Im Vergleich der Verteilung der Kodierungen in den Hauptkategorien wird deutlich, dass die Probanden der EG stärker damit beschäftigt sind, sich zu orientieren als Inhalte in ihrer Bedeutung zu erfassen und zu elaborieren. Der Kalkulator der Commerzbank erfordert dementsprechend offenbar viele Orientierungsprozesse, die zu Lasten essentieller Lernprozesse gehen können.

Im Rahmen der Think-aloud-Kodierungen wurden die Aussagen zum Nichtverstehen bzw. zu Unsicherheiten einer ergänzenden inhaltlichen Analyse unterzogen (Forschungsfrage III). Die hier erfassten Kodierungen der Aussagen liefern Hinweise auf mögliche Fehlkonzepte und Fehler in der Informationsverarbeitung, die Fehlkonzepte begünstigen (Tab. 4).

Verständnisprobleme konnten insbesondere für die Konzepte Annuität, Zinsfestschreibung, anfängliche jährliche Tilgung, Bereitstellungszins, Eigenkapital, Eigentum und Wohneinheit identifiziert werden. Obwohl für diese Konzepte teils Erläuterungen im Darlehenskalkulator hinterlegt sind, verstehen nicht alle Proband*innen der EG deren Bedeutung korrekt. Unklarheiten entstanden auch hinsichtlich der Ermittlung des Darlehensbetrags. Daneben greifen Proband*innen, anstatt die Annuitätenrechnung als Verfahren zu nutzen, auf überschlägige Näherungsrechnungen zurück. Als Fehlkonzepte sind insbesondere der Bereitstellungszins, die Wohneinheiten und der anfängliche jährliche Tilgungssatz zu identifizieren. So wird z. B. der Bereitstellungszins als Strafzins für Sondertilgungen verstanden, die Wohneinheit mit der Anzahl der Räume gleichgesetzt und die anfängliche jährliche Tilgung als zusätzlich zur Annuität aufzubringender Tilgungsbetrag interpretiert. 
Tab. 4: Fehlkonzepte

\begin{tabular}{lllll}
\hline Fehlkonzepte & $\begin{array}{l}\text { Häufigkeit } \\
\text { absolut }\end{array}$ & $\begin{array}{l}\text { Häufigkeit } \\
\text { relativ }\end{array}$ & $\begin{array}{l}\text { KG } \\
(\mathrm{n}=6)\end{array}$ & $\begin{array}{l}\text { EG } \\
(\mathrm{n}=12)\end{array}$ \\
\hline $\begin{array}{l}\text { Annuität (Zins und } \\
\text { Tilgung) }\end{array}$ & 10 & 16.67 & 7 & 3 \\
$\begin{array}{l}\text { Darlehensbetrag } \\
\begin{array}{l}\text { Zinsfestschreibung } \\
\text { (Zinsbindung) }\end{array}\end{array}$ & 10 & 16.67 & 3 & 7 \\
$\begin{array}{l}\text { Bereitstellungszins } \\
\text { (-freie Zeit) }\end{array}$ & 5 & 11.67 & 2 & 5 \\
Näherungsrechnung & 5 & 8.33 & 0 & 5 \\
$\begin{array}{l}\text { Prozentrechnungs- } \\
\text { fehler }\end{array}$ & 5 & 8.33 & 5 & 0 \\
Wohneinheit & 5 & 8.33 & 4 & 1 \\
Eigentum & 4 & 8.33 & 0 & 5 \\
$\begin{array}{l}\text { Anfängliche jährliche } \\
\text { Tilgung }\end{array}$ & 2 & 6.67 & 0 & 4 \\
Bauart & 2 & 3.33 & 1 & 1 \\
Eigenkapital & 2 & 3.33 & 0 & 2 \\
Erbbaurecht & 1 & 3.33 & 2 & 0 \\
Frei verfügbares & 1 & 1.67 & 0 & 1 \\
Einkommen & 1 & 1.67 & 0 & 1 \\
Nebenkosten & 60 & 1.67 & 0 & 1 \\
Summe & & & 24 & 36 \\
\hline & & & & \\
\hline
\end{tabular}

Quelle: eigene Abbildung

Konsequenzen für die Förderung von Finanzkompetenz

Die Ergebnisse der Studie zeigen, dass das Explorieren des ausgewählten Darlehenskalkulators nicht zu besserem Verständnis in Bezug auf die darin enthaltenen Konzepte und Zusammenhänge führt. Zudem fördert die Webseite zu wenig relevante kognitive Aktivitäten, wie das Evaluieren von Informationen 
und Bedeutungen sowie das Ableiten von Konsequenzen; es entstehen oder es manifestieren sich einige Fehlkonzepte, die Entscheidungen beeinflussen können. Online verfügbare Informationen zu Finanzprodukten tragen damit nicht zwangsläufig zu einer Förderung von Finanzkompetenz bei.

Um informelles Lernen aus Online-Informationen zu Finanzprodukten wirksam zu gestalten, sollten diese Informationen und deren Darbietung Denkprozesse höherer Ordnung stimulieren (Mayer 2009; Damnik et al. 2013). Individuen sollten dabei unterstützt werden, neue Informationen zu selektieren, $\mathrm{zu}$ organisieren und in bestehende Wissensstrukturen zu integrieren. Dazu sollten die relevanten Konzepte und Zusammenhänge (z. B. zur Annuitätenrechnung) präsentiert und auf weniger relevante Informationen verzichtet werden. Weitere Ansatzpunkte für die Gestaltung der Webseiten und die lernförderliche Präsentation von Informationen liefern die Prinzipien multimedialen Lernens (Mayer 2017), die in Folgestudien umgesetzt und auf ihre Wirkung hin überprüft werden sollten.

\section{Diskussion}

Die Ausführungen zur Messung von Finanzkompetenz von Förster und Kraitzek haben die Bedeutung von Messverfahren, die nicht nur Single- oder Multiple-Choice-Items verwenden und nicht ausschließlich auf Faktenwissen zielen, verdeutlicht. Weiterhin haben die beiden Studien von Wuttke, Siegfried und Aprea sowie Hommel und Fürstenau gezeigt, dass der Einsatz von komplexen Verfahren zur Messung von Finanzkompetenz einen Mehrwert gegenüber den genannten bestehenden Verfahren hat. Konkret lässt sich benennen, dass die Studien nicht nur Faktenwissen, sondern auch Entscheidungsverhalten berücksichtigen. Hinzu kommt, dass sie jeweils einen weiteren Schwerpunkt berücksichtigen. Dieser betrifft in der Studie von Wuttke, Siegfried und Aprea den Gender Gap, in der Studie von Hommel und Fürstenau das informelle Lernen sowie darauf bezogene kognitive Prozesse und Fehlkonzepte. Einzelne Aspekte der Beiträge sollen im Folgenden noch etwas näher betrachtet werden.

Aus dem Beitrag von Förster und Kraitzek geht hervor, dass die meisten Verfahren im internationalen und deutschen Kontext den Fokus auf kognitive Dispositionen im Assessment finanzieller Kompetenz legen. Deutlich wird jedoch auch, dass in neueren Entwicklungen eine ausgeprägtere Kompetenzorientierung zu finden ist, die sich durch einen stärkeren Situations- und lebensweltlichen Bezug der Aufgaben sowie komplexere Fragestellungen auszeichnen. Dabei kann diskutiert werden, wie komplex die Situation und der Problemlöseprozess für die Probanden in neuen Assessmentverfahren ausgestaltet werden sollten, um möglichst authentische Entscheidungsprozesse zu simulie- 
ren. Hier sollte die Komplexität der Aufgaben und der Situationsbeschreibungen weiter erhöht werden, um eine höhere ökologische Validität zu erzielen und der Realität noch besser gerecht zu werden. Förster und Kraitzek validieren hier in einem nächsten Schritt gerade die Lösung komplexer, alltagsnaher Problemszenarien im Finanzkontext, die aus mehreren Seiten realer Informationen (z. B. Aktienkurse, Anlagebedingungen verschiedener Produkte, etc.) bestehen. Schüler*innen müssen hier u. a. wichtige von unwichtigen Informationen abgrenzen und ihre Entscheidung fachlich begründen.

Die Studie von Wuttke, Siegfried und Aprea hat gezeigt, dass es zwei Testbereiche gibt, in denen weibliche Probanden tendenziell oder signifikant besser abschneiden als männliche Probanden. Es gibt jedoch wenige Studien, die auch einen Leistungsvorsprung weiblicher Teilnehmer bestätigen (z. B. Hill \& Asarta 2016; Jang et al. 2014; Walstad et al. 2010). Die wohl bekannteste ist hier die aktuelle PISA-Studie, gemäß der Schülerinnen in Ländern, wie Spanien, Polen oder Litauen, signifikant bessere Leistungen im Financial Literacy Tool erzielen als Schüler (OECD 2017a). Dennoch stellt sich das Phänomen des Gender Gaps in einzelnen Studien ganz unterschiedlich dar, und es wird eine Reihe an Hintergrundinformationen herangezogen, um den Gender Gap zu klären. Dazu gehören Unterschiede in individuellen Merkmalen zwischen Männern und Frauen, Unterschiede in sozio-ökonomischen Merkmalen, aber auch länderspezifische Merkmale oder Unterschiede in Lerngelegenheiten, die Männer und Frauen nutzen, um etwas über Finanzen in Erfahrung zu bringen (Bucher-Koenen et al. 2017). Hier stellt sich die Frage, ob noch weitere Hintergrundvariablen in die Studie mit einbezogen werden können, um nähere Informationen über den Vorteil für weibliche Probanden zu erhalten. Weiterhin wird das Aufgabenformat als Erklärungsfaktor für den Gender Gap herangezogen, da Ergebnisse zeigen, dass vor allem männliche Lernende bei Multipleoder Single-Choice-Items bevorteilt werden. Im Gegenzug stellt sich die Frage, ob es gegebenenfalls gerade die SJT sind, die Vorteile für weibliche Probanden mit sich bringen oder ob weitere Hintergrundvariablen zu berücksichtigen sind, um den Vorteil für diese Gruppe zu erklären. Um diese Frage näher zu beleuchten, ist der zentrale Prädiktor das Informations- und Kaufverhalten bzw. die Fähigkeit, Bedürfnisse aufzuschieben, näher zu untersuchen. Es ist zu klären, ob es deskriptive Analysen zu der eingesetzten Skala gibt, die Unterschiede zwischen Männern und Frauen zeigen und insofern den Gender Gap erklären könnten. Gleichzeitig wird deutlich, dass die Berücksichtigung des Prädiktors Informations- und Kaufverhalten bzw. Bedürfnisaufschub in den Regressionsanalysen eine Auswirkung auf den Gender Gap hat. Dies kann Anlass sein, in Folgestudien weitere Faktoren aufzunehmen, die mit dem Kaufverhalten und Bedürfnisaufschub in Verbindung stehen. Vorstellbar sind in diesem Zusammenhang Skalen, die Einstellung zu Geld, zu nachhaltigem Konsum oder zum reflektierten Umgang mit Werbung messen. Dabei könnten sich Unterschiede zwischen Männern und Frauen zeigen, die weiter zur Erklärung 
des Gender Gaps dienen. Das regressionsanalytische Vorgehen von Wuttke, Siegfried und Aprea ließe sich folglich um weitere Hintergrundvariablen erweitern, zusätzlich könnte die Berücksichtigung von Interaktionseffekten eine noch differenziertere Analyse des Gender Gaps ermöglichen. Auch eine Erweiterung der Stichprobe bspw. um andere Alterskohorten ist denkbar.

Die Studie von Hommel und Fürstenau hat gezeigt, dass die Exploration von Internetseiten das informelle Lernen und die Kompetenzentwicklung kaum unterstützen. Dadurch wird die Bedeutung, Finanzkompetenz in formalen Settings zu fördern oder informelles und formales Lernen zu koppeln, unterstrichen. Es ist jedoch zu berücksichtigen, dass das Ergebnis informellen Lernens unterschiedliche Ursachen haben kann: Einerseits sind die Webseiten zwar zur Informationsbeschaffung gedacht, aber Banken verfolgen vermutlich nicht in erster Linie die Intention, umfassende Lernprozesse anzustoßen. Möglicherweise unterscheiden sich diesbezüglich jedoch Direktbanken von traditionellen Banken. Hinzu kommt, dass die Webseiten auf den ersten Blick wenig kognitiv aktivierend wirken. Vielmehr wird eine Informationsfülle dargeboten, die im Zweifel sogar zur Überforderung der Nutzer*innen führen kann. Dies gilt insbesondere dann, wenn Anwender*innen über mangelnde fachliche oder digitale Kompetenzen verfügen. $\mathrm{Zu}$ überlegen wäre für weitere ähnlich gelagerte Studien, eine Skala zu digitalen Kompetenzen einzusetzen oder den Umgang mit den Webseiten, z. B. über Log-Files, mit zu erfassen. Auch eine Erweiterung der Stichprobe über Studierende hinaus wäre denkbar, um zu analysieren, ob sich vergleichbare Ergebnisse auch für andere Personengruppen zeigen. Möglich wäre beispielsweise ein Zusammenhang zwischen informellen Lernprozessen und der Intention, Eigentum zu erwerben. $\mathrm{Zu}$ berücksichtigen ist jedoch auch, dass die Webseiten die persönliche Beratung nicht ersetzen, sondern die Kunden gerade zu einem persönlichen Gespräch mit einem Berater oder einer Beraterin motivieren sollen. Eine Stärke der Studie kann in dem komplexen Messverfahren und dem umfangreichen Datenpool gesehen werden. Insbesondere die Daten zu den Fehlkonzepten liefern zentrale Implikationen für formale Lernprozesse. Einerseits zeigen die Ergebnisse, dass es Konzepte, wie Zins, Tilgung oder Zinsfestschreibung, sind, die Studierende nicht richtig verstehen. Diese Themen könnten von der Fachwissenschaft und der Fachdidaktik noch einmal aufgegriffen werden, um ein Conceptual Understanding oder ein Conceptual Change bei Studierenden anstoßen zu können.

Insgesamt lässt sich feststellen, dass im Symposium der Stand zum Assessment aufgearbeitet wurde und die vorgestellten Studien Hinweise darauf geben, wie die Messung von Finanzkompetenz verbessert und die Forschung weiterentwickelt werden kann, sei es zum Gender Gap, sei es zum informellen Lernen oder zu Fehlkonzepten. 


\section{Literatur}

Aprea, C., Wuttke, E., Leumann, S. \& Heumann, M. (2015). Kompetenzfacetten von Financial Literacy: Sichtweisen verschiedener Akteure. In J. Seifried, S. Seeber \& B. Ziegler (Hrsg.), Jahrbuch der berufs- und wirtschaftspädagogischen Forschung. Schriftenreihe der Sektion Berufs- und Wirtschaftspädagogik der Deutschen Gesellschaft für Erziehungswissenschaft (DGfE) (S. 11-22). Opladen: Barbara Budrich.

Aprea, C. \& Wuttke, E. (2016). Financial literacy of adolescent and young adults: setting the course for a competence-oriented assessment approach. In C. Aprea, E. Wuttke, K. Breuer, N.K. Keng, P. Davies, B. GreimelFuhrmann \& J. Lopus (eds.), International Handbook of Financial Literacy (pp. 397-414). Singapore: Springer.

Aprea, C., Wuttke, E., Breuer, K., Keng, N.K., Davies, P., Greimel-Fuhrmann, B. \& Lopus, J. (2016). Financial literacy in the twenty-first century: An introduction to the International Handbook of Financial Literacy. In C. Aprea, E. Wuttke, K. Breuer, N.K. Keng, P. Davies, B. Greimel-Fuhrmann \& J. Lopus (eds.), International Handbook of Financial Literacy (pp. 14). Singapore: Springer.

Barry, D. (2014). Die Einstellung zu Geld bei jungen Erwachsenen. Eine Grundlegung aus wirtschaftspädagogischer Sicht. Wiesbaden: Springer VS.

Bender, N. \& Barry, D. (2013). Adaption of the TRSQ for Financial Behavior. In K. Beck \& O. Zlatkin-Troitschanskaia (eds.), From Diagnostics to Learning Success. Proceedings in Vocational Education and Training (pp. 213-224). Rotterdam: Sense.

Breuer, K. (1999). Befähigung zur Selbstregulation in der beruflichen Aus- und Weiterbildung (Fragebogen zu Lernen und Denken). Deutsche Fassung des Trait Self-Regulation Questionnaire (TSRQ) nach O'Neil \& Herl. Unveröffentlichter Fragebogen. Mainz.

Bucher-Koenen, T., Lusardi, A., Alessie, R., van Rooij, M. (2017). How Financially Literate Are Women? An Overview and New Insights. Journal of Consumer Affairs, 51(2), 255-238.

Cerasoli C. P., Alliger G. M., Donsbach, J. S., Mathieu, J. E., Tannenbaum, S. I. \& Orvis, K. A. (2018) Antecedents and Outcomes of Informal Learning Behaviors: A Meta-Analysis. Journal of Business Psychology, 33, 203230

Chi, M. T. H., Bassok, M., Lewis, M. W., Reimann, P. \& Glaser, R. (1989). Self-Explanations: How Students Study and Use Examples in Learning to Solve Problems. Cognitive Science, 13, 145-182.

Council for Economic Education CEE (2013). National Standards for Financial Literacy. New York.

Damnik, G., Proske, A., Narciss, S. \& Körndle, H. (2013). Informal Learning with Technology: The Effects of Self-Constructing Externalizations. The Journal of Educational Research, 106, 431-440. 
Dennen, V. P. \& Wang, M. (2002). The Keyboard-Based Job Coach: Informal Learning via the Internet. Advances in Developing Human Resources, 4(4), 440-450.

Eitel, A., Dersch, A.-S. \& Renkl, A. (2019). Wissenschaftliche Annahmen und Fehlkonzepte angehender Lehrkräfte über das Lernen mit Multimedia. Unterrichtswissenschaft, 47, 451-474.

Förster, M., Happ, R. \& Molerov, D. (2017). Using the U.S. Test of Financial Literacy in Germany - Adaption and Validation. Journal of Economic Education, 48(2), 123-135.

Fürstenau, B., Hommel, M., Leopold, C., Ponce, H. \& López, M. (2016). Analysis of Banks' Online Information Regarding Mortgages as a Basis for Financially Literate Decision-Making in First-Time Homebuying. In E. Wuttke, J. Seifried \& S. Schumann (eds.), Economic Competence and Financial Literacy of Young Adults. Status and Challenges (pp. 63-79). Opladen u. a.: Barbara Budrich.

Fürstenau, B. \& Hommel, M. (2018). Contribution of bank webpages to the development of young adults' financial competence about mortgages. Empirische Pädagogik, 32(3/4), 434-459.

Fürstenau, B. \& Hommel, M. (2019). Developing financial competence about mortgage loans by informal learning using banks' online calculators. Empirical Research in Vocational Education and Training, 11(10), 1-33.

Geiger, J.-M., Meretz, U. \& Liening, A. (2016). Systematisierung deutschsprachiger Studien zur Kompetenzerfassung von financial literacy. Zeitschrift für ökonomische Bildung, 5, 72-93.

Greimel-Fuhrmann, B. \& Silgoner, M. (2018). Analyzing the Gender Gap in Financial Literacy. International Journal for Infonomics (IJI), 11(3), $1779-1787$.

Grohs-Müller, S. (2018). Schüler/innen und ihr Umgang mit Geld. Dissertation, Wirtschaftsuniversität Wien.

Hausmann, V. \& Retzmann, T. (2014). US-amerikanische Tests für die Ökonomische Bildung - Ein Modell für Deutschland? In T. Retzmann (Hrsg.), Ökonomische Allgemeinbildung in der Sekundarstufe I und Primarstufe (S. 330-345). Schwalbach/Ts.: Wochenschau Wissenschaft.

Happ, R. \& Förster, M. (2019). The relationship between migration background and knowledge and understanding of personal finance of young adults in Germany. International Review of Economics Education, 30(1), $1-14$.

Hill, A. T. \& Asarta, C. J. (2016). Gender and student achievement in personal finance: Evidence from keys to financial success. In C. Aprea, E. Wuttke, K. Breuer, N.K. Keng, P. Davies, B. Greimel-Fuhrmann \& J. Lopus (eds.), International Handbook of Financial Literacy (pp. 545-567). Singapore: Springer.

Holland, A. A. (2019). Effective principles of informal online learning design: A theory-building metasynthesis of qualitative research. Computers $\&$ Education, 128, 214-226. 
Hommel, M., Fürstenau, B., Leopold, C., Ponce, H. \& López, M. (2017). Beitrag von Banken-Webseiten zur Entwicklung der Finanzkompetenz potentieller Darlehensnehmer/innen über Baufinanzierungen. In J. Seifried, S. Seeber \& B. Ziegler (Hrsg.), Jahrbuch der berufs- und wirtschaftspädagogischen Forschung 2017. Schriftenreihe der Sektion Berufs- und Wirtschaftspädagogik der Deutschen Gesellschaft für Erziehungswissenschaft (DGfE) (S. 97-111). Opladen: Barbara Budrich.

Jang, K., Hah, J. \& Park, H. (2014). Comparison of financial literacy between Korean and U.S. High School Students. International Review of Economics Education, 16, 22-38.

Jerusalem, M. \& Schwarzer, R. (1999). Allgemeine Selbstwirksamkeit. In R. Schwarzer \& M. Jerusalem (Hrsg.), Skalen zur Erfassung von Lehrer- und Schülermerkmalen (S. 13-14) Berlin: Freie Universität.

Kahmann, J. (2014). Entwicklung und Validierung eines Situational Judgement Tests (SJT) zur Erfassung sozialer Kompetenzen von Studienplatzbewerbern und -interessenten der Human und Zahnmedizin. Dissertation. Heidelberg.

Kraitzek, A. (in Vorbereitung). Utilization of the U.S. Test of Financial Knowledge in Germany - Adaption and Validation. Annual Meeting of the American Educational Research Association (AERA), San Francisco.

Krippendorf, K. (2012). Content Analysis: An Introduction To Its Methodology. London: Sage.

Lindner, M. A., Strobel, B. \& Köller, O. (2015). Multiple-Choice-Prüfungen an Hochschulen? Ein Literaturüberblick und Plädoyer für mehr praxisorientierte Forschung. Zeitschrift für Pädagogische Psychologie, 29(3-4), $133-149$.

Lusardi, A. \& Mitchell, O. S. (2009). How Ordinary Consumers Make Complex Economic Decisions: Financial Literacy and Retirement Readiness. NBER Working Paper, No. 15350, Online: https://www.nber.org/papers/w15350.pdf (03.12.2019).

Lusardi, A. \& Mitchell, O. S. (2011). Financial Literacy around the world: an overview. Journal of Pension Economics and Finance, 10(4), 497-508.

Lusardi, A. \& Mitchell, O. S. (2014). The Economic Importance of Financial Literacy: Theory and Evidence. Journal of Economic Literature, 52(1), 544.

Maier, M., Rothmund, T., Retzbach, A., Otto, L \& Besley, J. C. (2014) Informal Learning Through Science Media Usage. Educational Psychologist, 49(2), 86-103.

Mayer, R. E. (2009). Multimedia learning ( $2^{\text {nd }}$ ed.). New York: Cambridge University Press.

Mayer, R. E. (2017). Using multimedia for e-learning. Journal of Computer Assisted Learning, 33, 403-423.

Mayring, P. (2015). Qualitative Inhaltsanalyse: Grundlagen und Techniken (12. Aufl.). Weinheim: Beltz.

McDaniel, M. A., Hartmann, N. S., Whetzel, D. L. \& Grubb, W. L. (2007). Situational judgment tests, response instructions, and validity: A metaanalysis. Personnel Psychology, 60, 63-91. 
OECD (2013). PISA 2012 Assessment and Analytical Framework: Mathematics, Reading, Science, Problem Solving and Financial Literacy, OECD Publishing. Online: http://dx.doi.org/10.1787/9789264190511-en (28.11.2019).

OECD (2017a). PISA 2015 Results. Students 'Financial Literacy. (Volume VI). Paris: OECD Publishing.

OECD (2017b). G20/OECD INFE report on adult financial literacy in G20 countries. Online: http://www.oecd.org/daf/fin/financial-education/G20OECD-INFE-report-adult-financial-literacy-in-G20-countries.pdf (28.11.2019).

Prenzel, M., Kristen, A., Dengler, P., Ettle, R. \& Beer, T. (1996). Selbstbestimmt motiviertes und interessiertes Lernen in der kaufmännischen Erstausbildung. In K. Beck \& H. Heid (Hrsg.), Lehr-Lern-Prozesse in der kaufmännischen Erstausbildung. Wissenserwerb, Motivationsgeschehen und Handlungskompetenzen (S. 108-127). Stuttgart: Franz Steiner.

Ray, J. \& Najman, J. (1986). The Generaliziability of Deferment of Gratification. Journal of Social Psychology, 126, 117-119.

Reardon, S. F., Kalogrides, D., Fahle, E. M., Podolsky, A., \& Zárate, R. C. (2018). The Relationship between Test Item Format and Gender Achievement Gaps on Math and ELA Tests in 4th and 8th Grade. Educational Researcher, 47(5), 284-294.

Rosendorfer, T. (2000). Kinder und Geld. Gelderziehung in der Familie. Frankfurt a. M.: Campus.

Rudeloff, M. (2019). Der Einfluss informeller Lerngelegenheiten auf die Finanzkompetenz von Lernenden am Ende der Sekundarstufe I. Wiesbaden: Springer.

Saygin, P. O. (2019). Gender bias in standardized tests: evidence from a centralized college admissions system. Empirical Economics. https://doi.org/ 10.1007/s00181-019-01662-z.

Schürkmann, S. (2017). FILS: Financial Literacy Study. Validierung und Analyse einer schülerorientierten Financial Literacy. Oldenbourg: DeGruyter.

Schürkmann, S. \& Schuhen, M. (2013). Kompetenzmessung im Bereich financial literacy. Ergebnisse zum Umgang mit Online-Rechnern aus der FILSStudie. Zeitschrift für ökonomische Bildung, 1, 73-89.

Siegfried, C. \& Wuttke, E. (2019). Are Multiple Choice Items unfair? And if so, for whom? Citizenship, Social and Economics Education, 18(3), 123127.

Temme, D. \& Hildebrandt, L. (2008). Gruppenvergleiche bei hypothetischen Konstrukten - Die Prüfung der Ubereinstimmung von Messmodellen mit der Strukturgleichungsmethodik. Schriftenreihe Economic Risk SFB 649 Papers, Discussion Paper 2008-042.

Walstad, W. B, Rebeck, K. \& MacDonald, R. A. (2010). The effects of financial education on the financial knowledge of High School students. The Journal of Consumer Affairs, 44(2), 336-357.

Walstad, W. B. \& Rebeck, K. (2016a). Basic Finance Test: Examiner's Manual. New York: Council for Economic Education.

Walstad, W. B. \& Rebeck, K. (2016b). Test of Financial Knowledge: Examiner's Manual. New York: Council for Economic Education. 
Walstad, W. B. \& Rebeck, K. (2016c). Test of Financial Literacy: Examiner 's Manual. New York: Council for Economic Education.

Weinert, F. E. (2001). Concept of Competence: A Conceptual Clarification. In D. S. Rychen \& L. H. Salganik (Hrsg.), Defining and Selecting Key Competencies (pp. 45-65). Seattle, Toronto: Hogrefe and Huber.

Weinert, F. E. (2002). Vergleichende Leistungsmessung in Schulen - eine umstrittene Selbstverständlichkeit. In F. E. Weinert (Hrsg.), Leistungsmessungen in Schulen (S. 17-31). Weinheim: Beltz.

Werquin, P. (2010). Recognising Non-Formal and Informal Learning. Outcomes, Policies and Practices. OECD Publishing, Paris.

Winther, E. (2006). Motivation in Lernprozessen. Konzepte in der Unterrichtspraxis von Wirtschaftsgymnasien. Wiesbaden: DUV.

Wuttke, E. \& Aprea, C. (2018). A situational judgement approach for measuring young adults' financial literacy. Empirische Pädagogik, 32, 272-292.

Wuttke, E., Siegfried, C., \& Aprea, C. (in Vorbereitung). Measuring financial literacy with a Situational Judgement Test - do some groups really perform worse or is it the measuring instrument? 
Appendix: Übersicht über Messinstrumente zur Financial Literacy

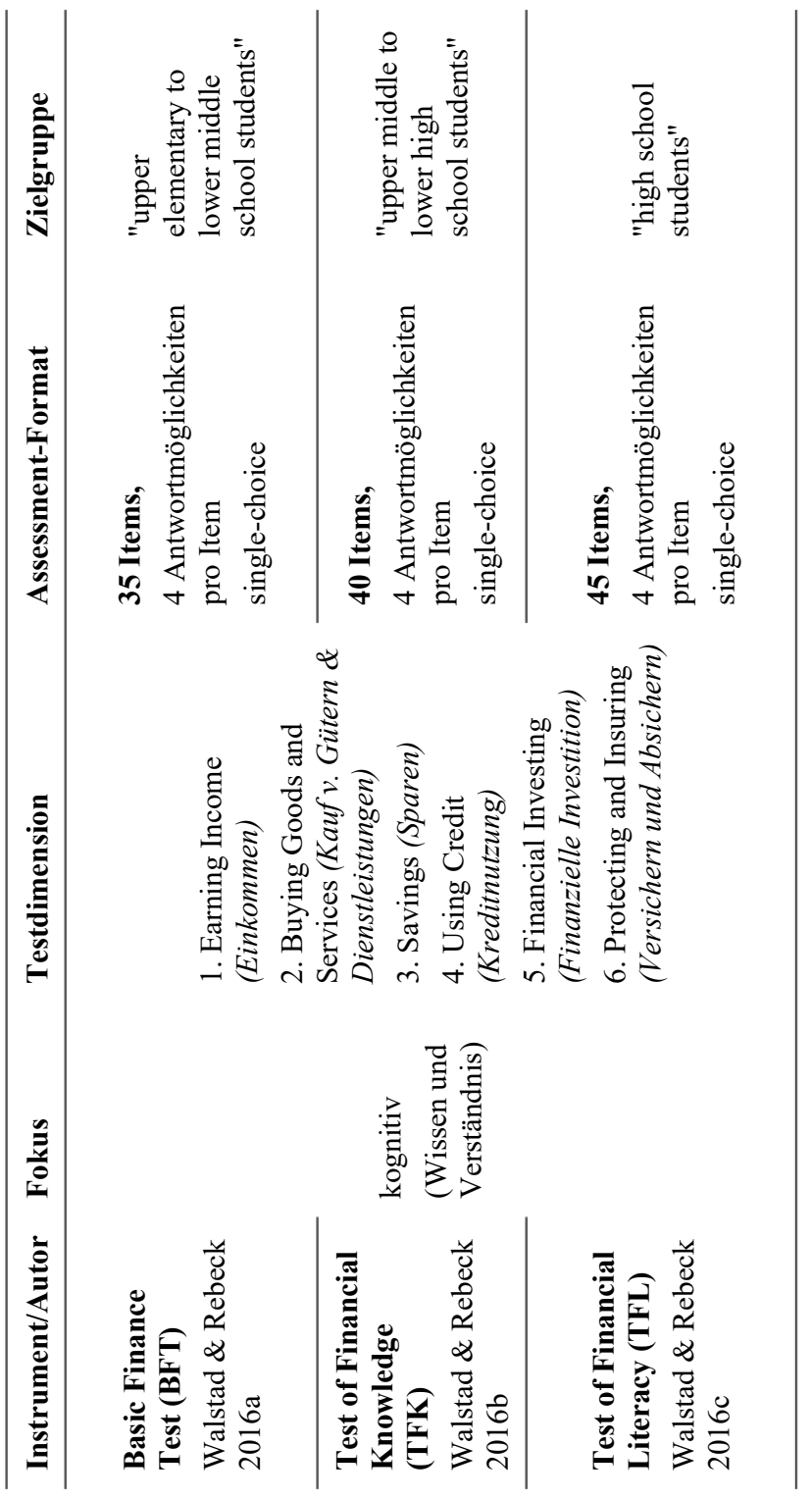




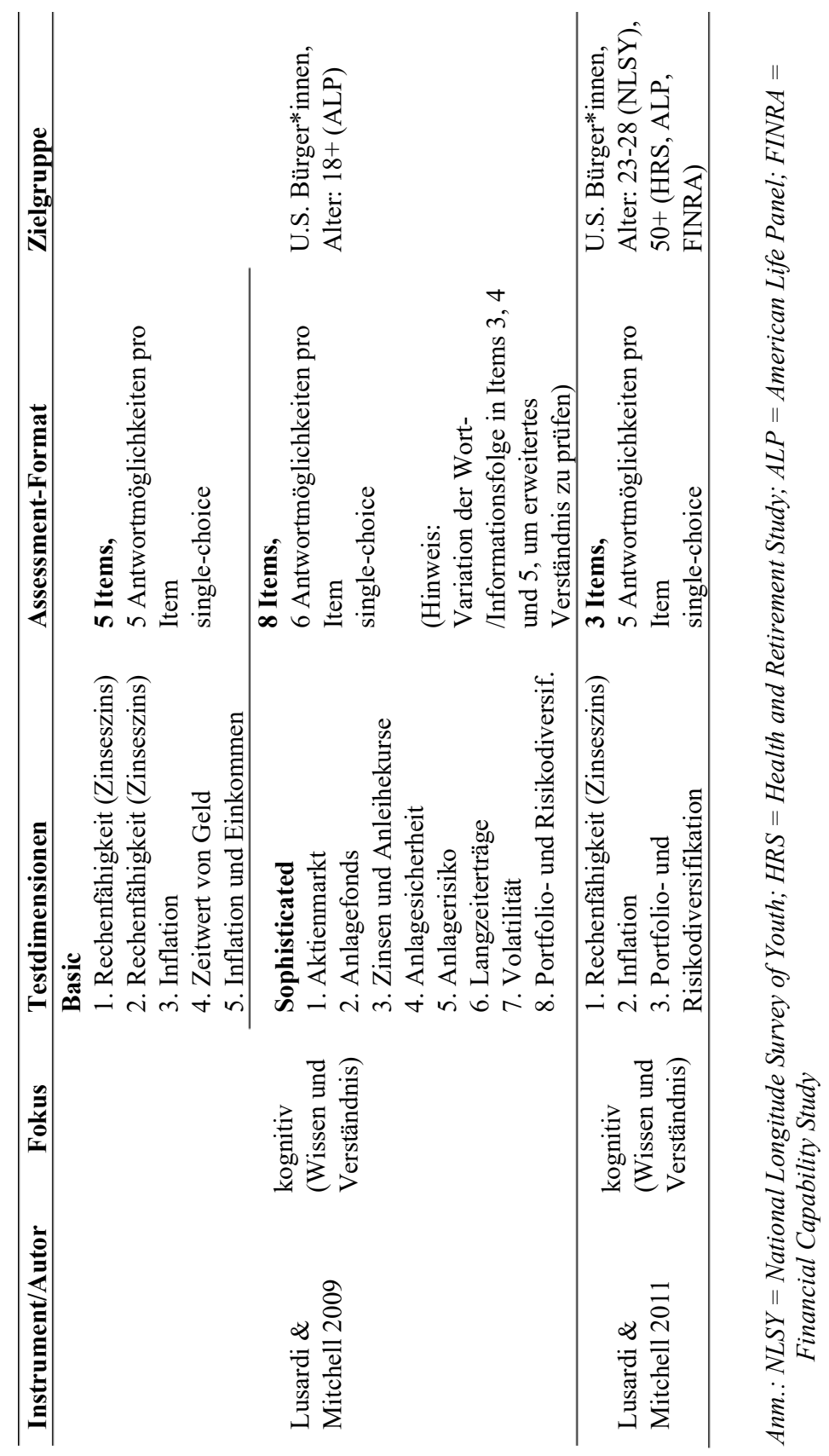




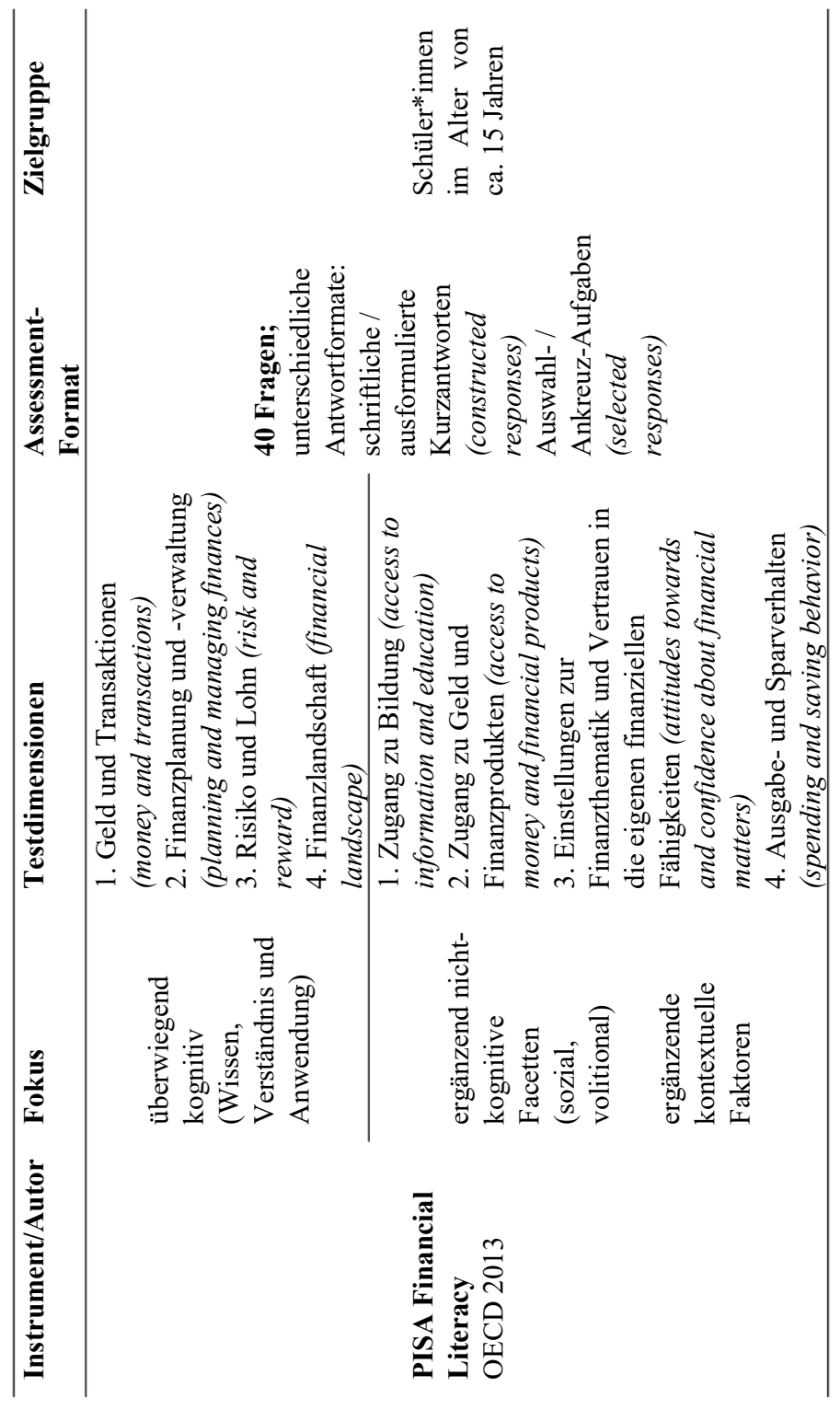




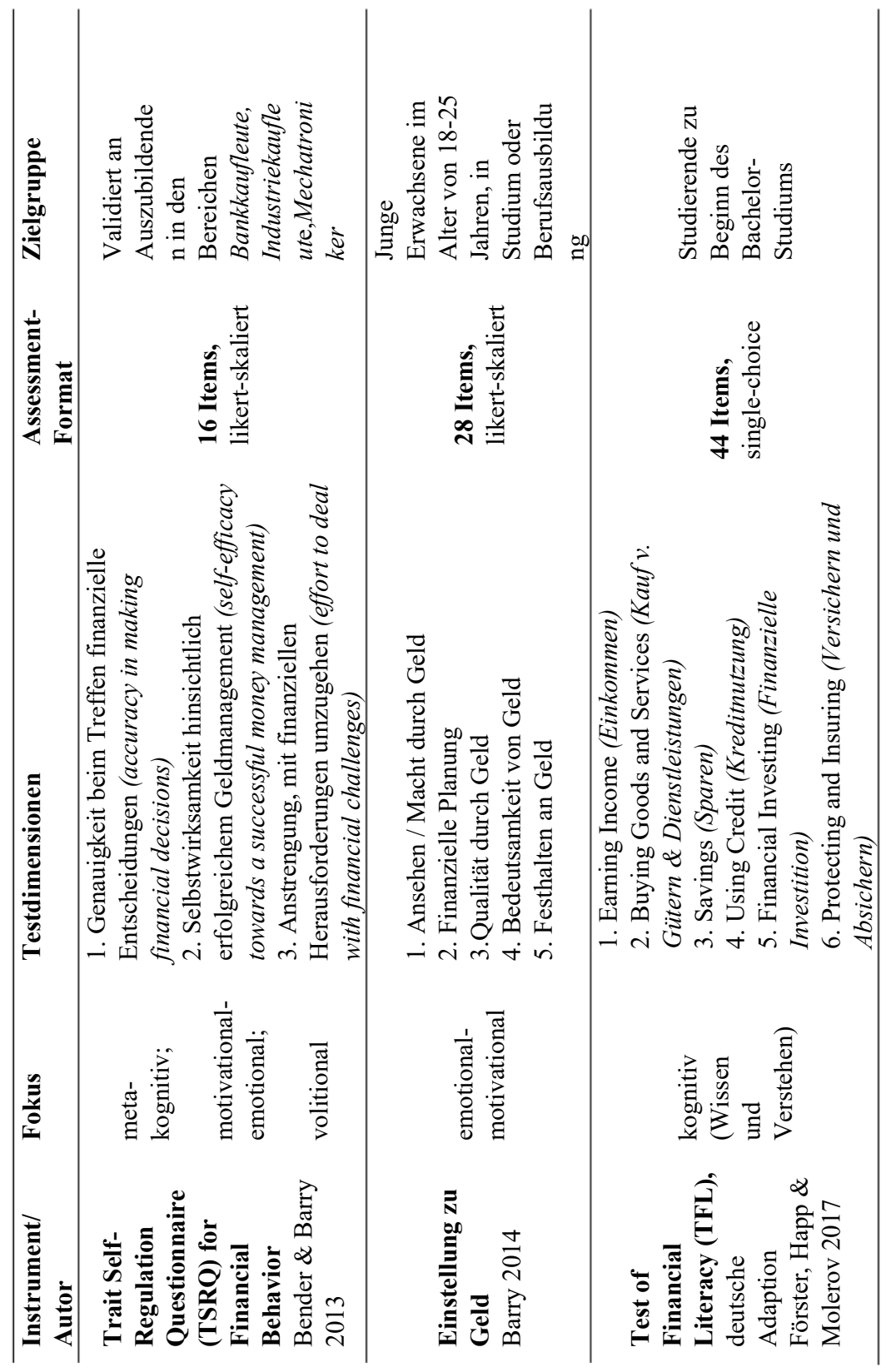




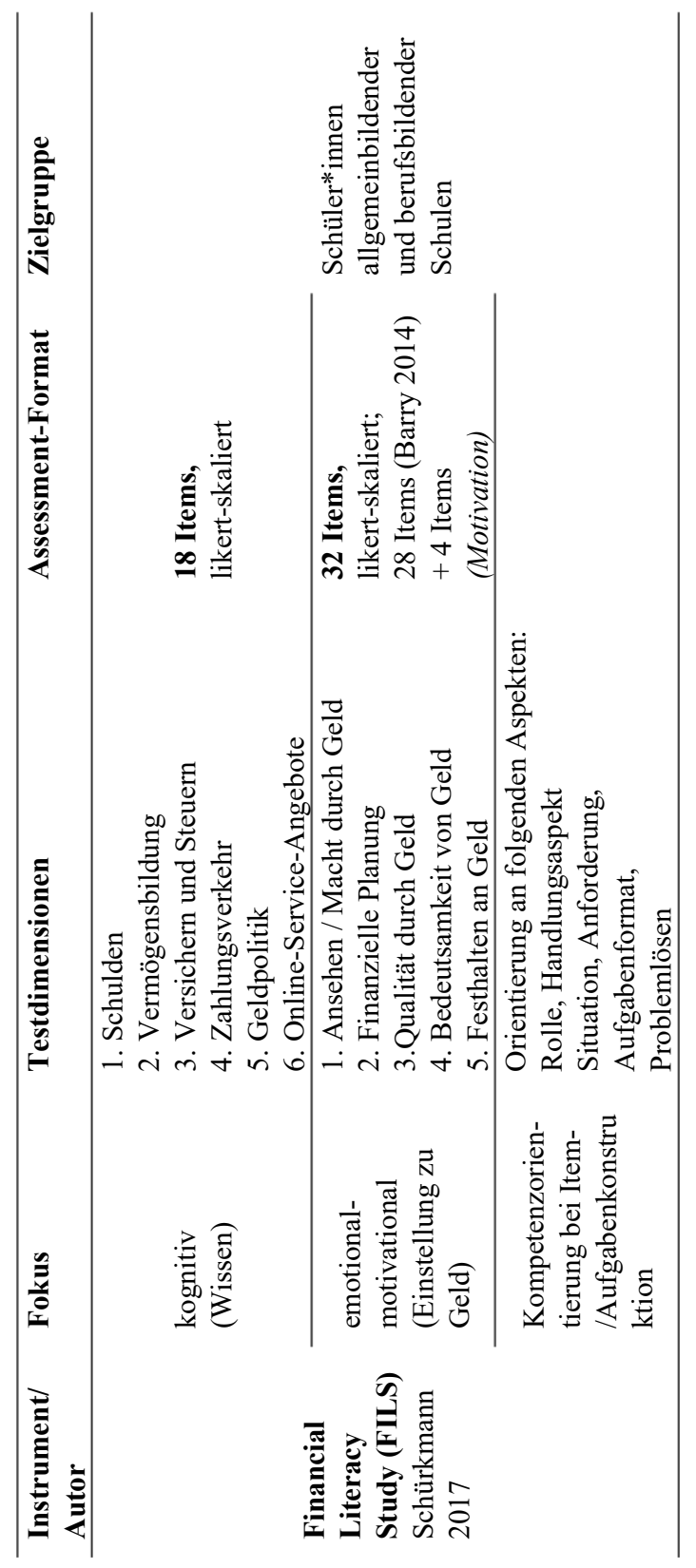




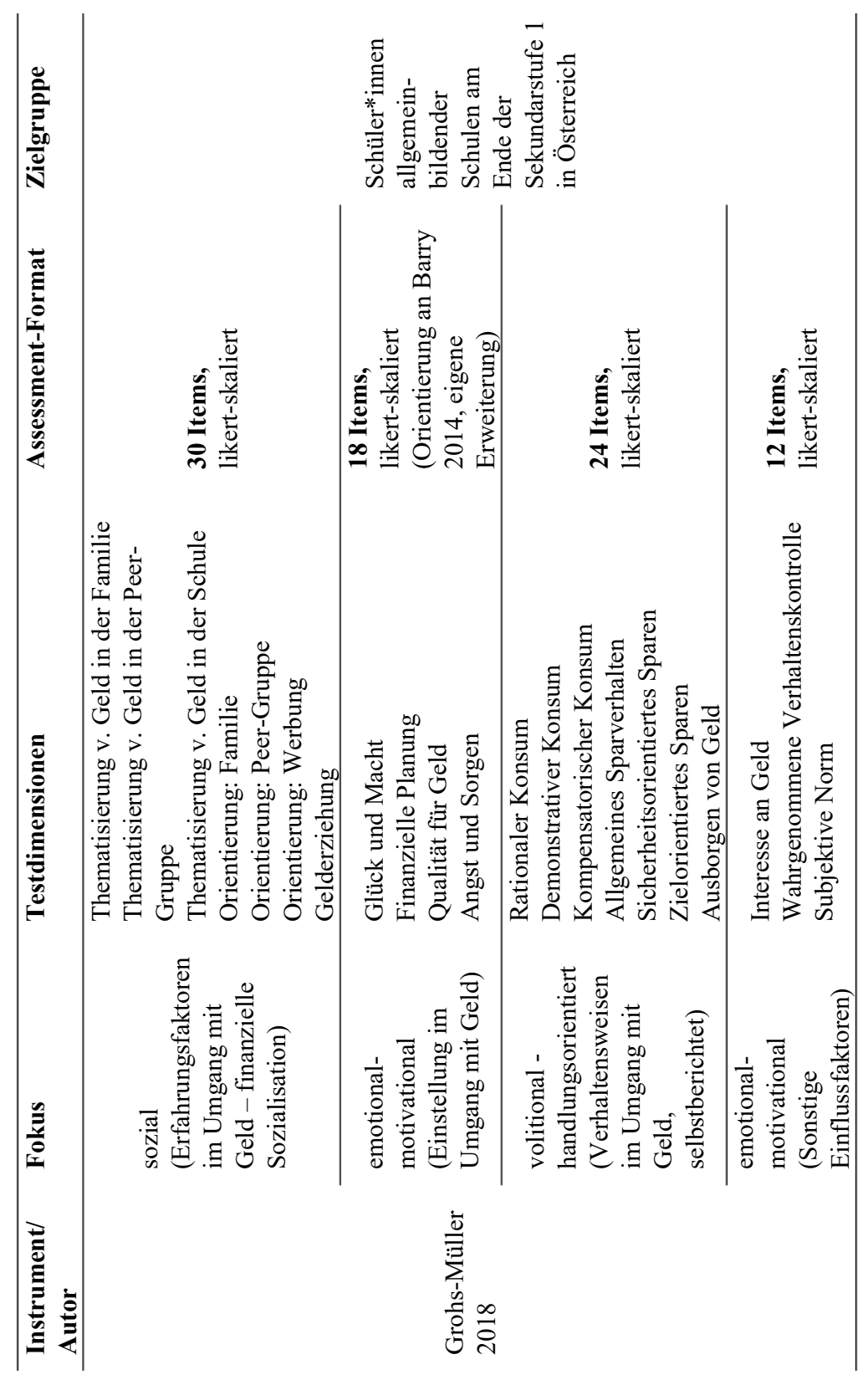




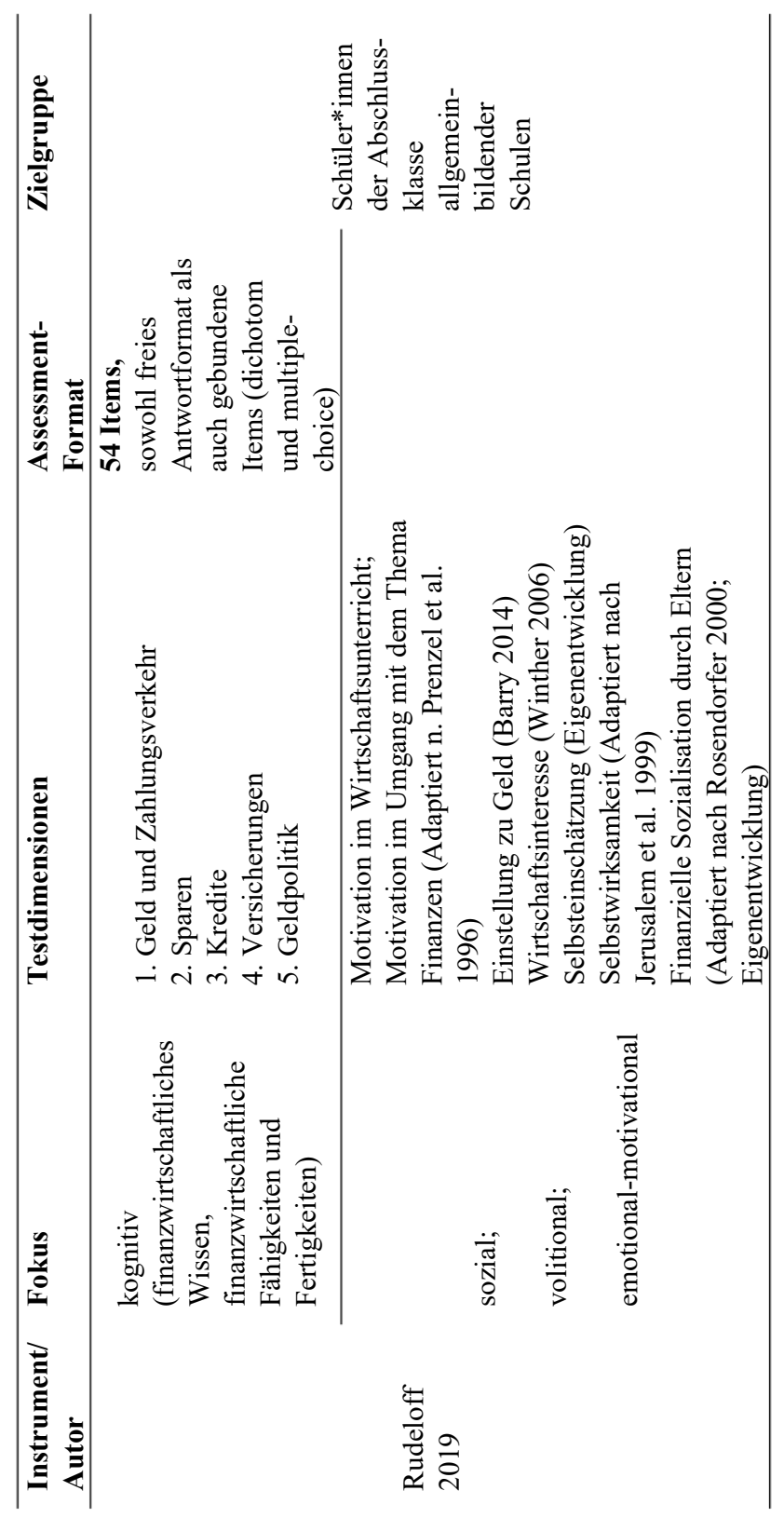




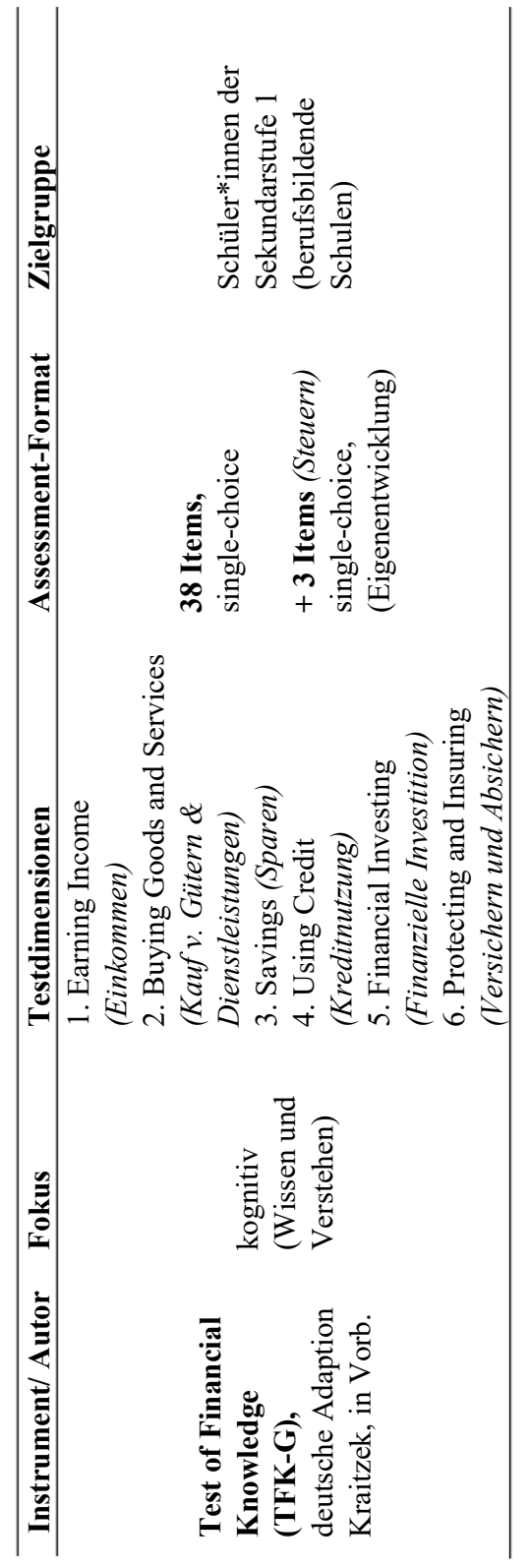

